\title{
CLOSURES OF CONJUGACY CLASSES IN CLASSICAL REAL LINEAR LIE GROUPS. II ${ }^{1}$
}

BY

DRAGOMIR Ž. DJOKOVIĆ

\begin{abstract}
By a classical group we mean one of the groups $G L_{n}(R), G L_{n}(C)$, $G L_{n}(H), U(p, q), O_{n}(C), O(p, q), S O^{*}(2 n), S p_{2 n}(C), S p_{2 n}(R)$, or $S p(p, q)$. Let $G$ be a classical group and $L$ its Lie algebra. For each $x \in L$ we determine the closure of the orbit $G \cdot x$ (for the adjoint action of $G$ on $L$ ). The problem is first reduced to the case when $x$ is nilpotent. By using the exponential map we also determine the closures of conjugacy classes of $G$.
\end{abstract}

0. Introduction. Let $G$ be a classical real linear Lie group (see $\$ 1$ for precise definition) and $L$ its Lie algebra. There are ten infinite series of such groups and we use index $j(1 \leqslant j \leqslant 10)$ to label these series. $G$ acts on $L$ via the adjoint representation and on itself by conjugation. The classification of orbits of $G$ in $L$ and the classification of conjugacy classes of $G$ are now well known; see for instance [1] and the references mentioned there. In this paper we determine the closure of an arbitrary orbit $\theta$ and the closure of an arbitrary conjugacy class $\mathcal{C}$. (The topological terms refer to the ordinary topology of $G$ and $L$.)

For complex groups this problem was solved by M. Gerstenhaber [5,6]. An independent proof for complex special linear groups was given by J. Dixmier [2], and for complex orthogonal and symplectic groups by W. Hesselink [7] and the author [3]. In fact the results of Gerstenhaber and Hesselink are more general since they consider classical groups over any algebraically closed field.

In our previous paper [4] on the same subject we have announced the main result of the present paper. For the sake of convenience we repeat all the necessary definitions so that the reader does not need to consult [4] except for the proofs of Theorem 1 (§2), Theorem 2 (§3), and the necessity part of Theorem 5 (\$7).

The two problems, closures of orbits and closures of conjugacy classes, are closely related. We give a full treatment to the first problem while the second is treated in a cavalier fashion in $\S 13$. In this introduction we shall comment only on the first problem.

In $\$ 2$ we state the Centralizer Theorem (Theorem 1) which asserts that the centralizer $C_{G}(x)$ in $G$ of a semisimple element $x \in L$ is a direct product of classical groups. The direct factors of $C_{G}(x)$ may belong to different series of classical groups.

Received by the editors May 5, 1980 and, in revised form, January 22, 1981.

1980 Mathematics Subject Classification. Primary 20G20; Secondary 22E46, 22 E60.

Key words and phrases. Classical group, adjoint representation, orbit, conjugacy class, Young diagram.

${ }^{1}$ This work was supported in part by NSERC of Canada Grant A-5285. Presented at the Algebra 1980 Conference, Carbondale, Illinois on April 19, 1980 and at the Annual Summer Meeting of the Canadian Mathematical Society, Montreal, on June 2, 1980. 
Each factor is associated with an eigenvalue of $x$ and the type of a factor is uniquely determined by the type of $G$ and the corresponding eigenvalue of $x$ (see Table III). This theorem provides justification and motivation for our definition of classical groups given in $\S 1$.

In $\S 3$ we show that our problem can be reduced to the case of nilpotent orbits. (An orbit of $G$ in $L$ is nilpotent if it consists of nilpotent elements.) The reduction is based on Theorem 2 for whose proof we refer to our paper [4]. Let $x \in L$ and let $x=x_{s}+x_{n}$ be its Jordan decomposition. (This means that $x_{s}, x_{n} \in L, x_{s}$ is semisimple, $x_{n}$ is nilpotent, and $\left[x_{s}, x_{n}\right]=0$.) Theorem 2 asserts that if $y$ belongs to the closure of the orbit $G \cdot x$ then there exists an element $z$ in the closure of the orbit $C_{G}\left(x_{s}\right) \cdot x_{n}$ such that $x_{s}+z \in G \cdot y$. Hence we can determine the closure of $G \cdot x$ provided we know how to find the closure of the orbit of $x_{n}$ under the group $C_{G}\left(x_{s}\right)$. Since $\left[x_{s}, x_{n}\right]=0$, the element $x_{n}$ belongs to the Lie algebra of $C_{G}\left(x_{s}\right)$. The reduction is completed now by invoking the Centralizer Theorem. Note that if we start with the classical group $G$ of type $j$ (i.e. belonging to the $j$ th series) our reduction requires us to consider the direct factors of $C_{G}\left(x_{s}\right)$ which may be of different type. For this reason all the classical groups should be treated simultaneously. We have not done so only in order to save space and to avoid duplication of the cases already treated in the literature. Hence in the very short $\S 4$ we dismiss the cases $j=1,2,3,5$ and 8 .

In $\$ 5$ we recall the definition of types for classical Lie algebras introduced by $\mathrm{N}$. Bourgoyne and R. Cushman [1]. We restrict ourselves to the cases $j=4,6,7,9,10$ and the nilpotent types. Let $V$ be a vector space and $f$ a form which defines the classical group $G$ of the $j$ th series (see $\S 1$ ). A type $\Delta$ is an equivalence class of triples $(V, f, x)$ where $x \in L$. Each type $\Delta$ can be written as a sum of indecomposable types and such a decomposition is unique. For details about types $\Delta$ and their properties we refer the reader to [1]. For each indecomposable nilpotent type we give a representative $(V, f, x)$. This is done mainly because our notation for polarized types $\Delta^{\cdot}$ differs from the notation in [1] and we wanted to avoid possible confusion. The types $\Delta_{m}^{+}$and $\Delta_{m}^{-}$which differ only in the superscript \pm can be distinguished by inequalities (5.3)-(5.5). Their importance in the rest of the paper cannot be overestimated.

In $\S 6$ we introduce combinatorial gadgets called chromosomes. They consist of genes which may be polarized or nonpolarized. In fact the set of chromosomes is a free commutative monoid on the set of genes. To each chromosome $X$ we assign its rank $r(X)=n$ and its signature $\operatorname{sig} X=(p, q)$ where $p$ and $q$ are either integers or half-integers such that $p \geqslant 0, q \geqslant 0$ and $p+q=n$. A submonoid of the set of chromosomes is called a variety. To each series of classical groups we assign a specific variety, say $\Phi_{j}$ to the $j$ th series (see Table IV in $\S 5$ ). We also define a partial order $\leqslant$ in each of the varieties $\Phi_{j}$.

In $\$ 7$ we establish (Lemma 4) a bijection between the nilpotent orbits of the classical group $G$ of the $j$ th series defined by $(V, f)$ and the set of chromosomes $X$ in $\Phi_{j}$ such that $\operatorname{sig} X=\operatorname{sig}(f)$. (See $\S 1$ for the definition of $\operatorname{sig}(f)$.) In the sequel we use the chromosomes as convenient labels which parametrize the nilpotent orbits. 
We also state here our main result (Theorem 5). It says that a nilpotent orbit $\theta_{1}$ is contained in the closure of the nilpotent orbit $\theta_{2}$ iff their corresponding chromosomes $X\left(\theta_{1}\right)$ and $X\left(\theta_{2}\right)$ satisfy the inequality $X\left(\theta_{1}\right) \leqslant X\left(\theta_{2}\right)$. This latter inequality refers to the partial order in $\Phi_{j}$ defined in $\S 6$. In Example 2 we illustrate the main theorem when $G=\operatorname{Sp}(2,2) \quad(j=10, \operatorname{sig}(f)=(2,2))$. The necessity of the condition $X\left(\theta_{1}\right) \leqslant X\left(\theta_{2}\right)$ was proved in [7] and we do not repeat the proof. The remainder of the paper is in essence the proof of the sufficiency of the above condition. It should be clear that this proof has to be based on the knowledge of the partial order $\leqslant$ in $\Phi_{j}$. (This is true in all the cases considered so far in the literature.)

In $\S 8$ we describe simple transformations of chromosomes of $\Phi_{j}$, say $X \rightarrow Y$, such that $X<Y$ and $\operatorname{sig} X=\operatorname{sig} Y$. We call these simple transformations mutations of chromosomes. When $j=4$ they are given by formulas (8.1)-(8.3), for $j=7,10$ by (8.4)-(8.8), and for $j=6,9$ by (8.9)-(8.17). Then we state our main theorem about partial orders $\leqslant$ in $\Phi_{j}$ (Theorem 6). It says that if $X \leqslant Y$ in $\Phi_{j}$ then there exists a chain of $\Phi_{j}$-mutations $X_{0} \rightarrow X_{1} \rightarrow \cdots \rightarrow X_{m}(m \geqslant 0)$ such that $X=X_{0}$ and $Y=X_{m}$. This theorem enables us to reduce the proof of Theorem 5 to a finite number of cases.

The proof of (the sufficiency part of) Theorem 5 occupies $\S \S 9-12$. $\S 9$ is of a preliminary nature. $\$ 10$ treats the case $j=4$ in detail. In $\S 11$ we treat the cases $j=7,10$ by reducing them to the case $j=4$. In $\S 12$ we treat the remaining two cases $j=6,9$. While in the case $j=4$ we have included all necessary computations we have not done this in the cases $j=6,9$ in order to keep $\$ 12$ of reasonable size.

As mentioned earlier $\$ 13$ deals with the problem of closures of conjugacy classes of $G$. We show that the problem can be reduced to the case of unipotent conjugacy classes and then the exponential map does the job.

The remaining $\S \S 14-17$ are devoted to the proof of Theorem 6 (see $\S 8$ ). $\S 14$ is of a preliminary nature. $\S 15$ deals with the case $j=4, \S 16$ with the cases $j=7,10$, and $\S 17$ with the cases $j=6,9$.

We use this occasion to correct several misprints in our previous paper [4]. The formula (12.3) should read:

$$
g_{\varepsilon}(m)+g_{-\varepsilon}(n) \rightarrow g_{-\varepsilon}(m-1)+g_{\varepsilon}(n+1), \quad m \leqslant n .
$$

In $\S 9$ in the description of the representative $(V, f, x)$ of $\Delta_{m}^{\varepsilon}(0,0), m$ odd, $j=7$ one should have $f\left(e_{r}, e_{s}\right)=-i \varepsilon \delta_{r+s, m}$ where $i=\sqrt{-1}$. Finally on p. 8 line 8 one should replace $x \in L$ by $x \in G$.

1. Classical groups. Let $V$ be a (right) $\mathbf{F}$-vector space where $\mathbf{F}=\mathbf{R}, \mathbf{C}$, or $\mathbf{H}$ and let $\operatorname{dim}_{\mathbf{F}} V=n<\infty$. (By $\mathbf{H}$ we denote the division algebra of real quaternions.) If $f$ : $V \times V \rightarrow \mathbf{F}$ is a nondegenerate hermitian form (including the case of symmetric forms if $\mathbf{F}=\mathbf{R}$ ) then we define the signature of $f$ to be the ordered pair $\operatorname{sig}(f)=\left(n_{+}, n_{-}\right)$where $n_{+}$(resp. $\left.n_{-}\right)$is the maximum dimension (over $\mathbf{F}$ ) of a positive (resp. negative) definite subspace of $V$. Thus $n_{+}+n_{-}=n$.

When $f$ is a nondegenerate skew-hermitian form (including the case of skew-symmetric forms if $\mathbf{F}=\mathbf{R})$ then we set $\operatorname{sig}(f)=(n / 2, n / 2)$. 
The words classical group will have a technical meaning which we now define. There are ten infinite series of classical groups and we use index $j(1 \leqslant j \leqslant 10)$ to refer to them. The first three series are the general linear groups

$$
G L(V) \cong G L_{n}(\mathbf{F})
$$

where $\mathbf{F}=\mathbf{C}$ for $j=1, \mathbf{F}=\mathbf{R}$ for $j=2$, and $\mathbf{F}=\mathbf{H}$ for $j=3$.

For the remaining seven series $V$ is equipped with a nondegenerate form $f$ : $V \times V \rightarrow \mathbf{F}$ and the corresponding classical group $G$ is the subgroup of $G L(V)$ which preserves $f$. The type of the form $f$ is specified in Table I for each of these series.

\begin{tabular}{rllll}
$j$ & $\mathbf{F}$ & $G$ & $f$ & $\operatorname{sig}(f)$ \\
\hline 4 & $\mathbf{C}$ & $U(k, n-k)$ & hermitian & $(k, n-k)$ \\
5 & $\mathbf{C}$ & $O_{n}(\mathbf{C})$ & symmetric & \\
6 & $\mathbf{R}$ & $O(k, n-k)$ & symmetric & $(k, n-k)$ \\
7 & $\mathbf{H}$ & $O^{*}(2 n)$ & skew-hermitian & $(n / 2, n / 2)$ \\
8 & $\mathbf{C}$ & $S p_{2 n}(\mathbf{C})$ & skew-symmetric & \\
9 & $\mathbf{R}$ & $S p_{2 n}(\mathbf{R})$ & skew-symmetric & $(n, n)$ \\
10 & $\mathbf{H}$ & $S p(k, n-k)$ & hermitian & $(k, n-k)$
\end{tabular}

\section{TABLE I}

Note that, according to our definition, the groups $S L_{n}(\mathbf{C}), S L_{n}(\mathbf{R}), S O_{n}(\mathbf{C})$, $S O(k, n-k), S U(k, n-k), S L_{n}(\mathbf{H})$ are not classical.

2. The centralizer theorem. From now on $G$ will denote a classical group belonging to the $j$ th series, $L$ its Lie algebra, and Ad: $G \rightarrow \operatorname{Aut}(L)$ the adjoint representation of $G$. For $a \in G$ and $x \in L$ we shall write $a \cdot x$ instead of $\operatorname{Ad}(a)(x)$. Thus $G \cdot x=\{a \cdot x: a \in G\}$ is the $G$-orbit in $L$ containing $x$. The centralizer $C_{G}(x)$ of $x \in L$ in $G$ is the subgroup of $G$ consisting of all elements $a$ such that $a \cdot x=x$. In this section we describe the structure of $C_{G}(x)$ for semisimple $x$. It turns out that this centralizer is a (finite) direct product of classical groups.

If $1 \leqslant j \leqslant 3$ we identify $L$ with the Lie algebra of all $\mathbf{F}$-linear transformations of $V$. If $4 \leqslant j \leqslant 10$ then we identify $L$ with the Lie algebra of all $\mathbf{F}$-linear transformations $x$ of $V$ which satisfy $f\left(x\left(v_{1}\right), v_{2}\right)+f\left(v_{1}, x\left(v_{2}\right)\right)=0$ for all $v_{1}, v_{2} \in V$.

Let $x \in L$ be arbitrary. If $\mathbf{F}=\mathbf{C}$ we define for $\zeta \in \mathbf{C}$ the subspace $V(\zeta, x)$ of $V$ to be

$$
V(\zeta, x)=\operatorname{Ker}(x-\zeta)^{\infty}
$$

i.e., it is the kernel of $(x-\zeta)^{k}$ for large $k$, say $k=n$. If $\mathbf{F}=\mathbf{R}$ of $\mathbf{H}$ we define for $\zeta \in \mathbf{C}$ the subspace $V(\zeta, x)$ of $V$ by

$$
V(\zeta, x)=\operatorname{Ker}\left(x^{2}-2 \operatorname{Re}(\zeta) x+|\zeta|^{2}\right)^{\infty}
$$


We say that $\zeta \in \mathbf{C}$ is an eigenvalue of $x \in L$ if $V(\zeta, x) \neq 0$.

It is well known that for $j \in\{5,6\}$ we have $V(\zeta, x) \perp V\left(\zeta^{\prime}, x\right)$ if $\zeta+\zeta^{\prime} \neq 0$, and for $j \in\{4,6,7,9,10\}$ we have $V(\zeta, x) \perp V\left(\zeta^{\prime}, x\right)$ if $\bar{\zeta}+\zeta^{\prime} \neq 0$. (The orthogonality is with respect to the form $f$.)

This suggests that we introduce the following definition:

$$
V^{\prime}(\zeta, x)= \begin{cases}V(\zeta, x) & \text { for } j \in\{1,2,3\} \\ V(\zeta, x)+V(-\zeta, x) & \text { for } j \in\{5,8\} \\ V(\zeta, x)+v(-\bar{\zeta}, x) & \text { for } j \in\{4,6,7,9,10\}\end{cases}
$$

Then we obtain a direct (and orthogonal if $j \geqslant 4$ ) decomposition

$$
V=\sum_{\zeta \in \Omega_{j}} V^{\prime}(\zeta, x)
$$

The set $\Omega_{j}$ is the subset of $\mathbf{C}$ specified by the conditions stated in Table II.

\begin{tabular}{clcc}
$j$ & \multicolumn{3}{c}{$\Omega_{j}$} \\
\hline 1 & $\mathbf{C}$ & & \\
2,3 & $\operatorname{Im} \zeta \geqslant 0$ & & \\
4 & $\operatorname{Re} \zeta \geqslant 0$ & & $\zeta \geqslant 0$ \\
5,8 & $\operatorname{Im} \zeta>0$ & or & $\operatorname{Im} \zeta \geqslant 0$
\end{tabular}

\section{TABLE II}

For $\zeta \in \mathbf{C}$ let $x_{\zeta}$ (resp. $\left.x_{\zeta}^{\prime}\right)$ be the restriction of $x$ to $V(\zeta, x)$ (resp. $V^{\prime}(\zeta, x)$ ). If $1 \leqslant j \leqslant 3$ let $G(\zeta, x)$ be the general linear group of the space $V(\zeta, x)$. If $4 \leqslant j \leqslant 10$ let $G(\zeta, x)$ be the group of all $\mathbf{F}$-linear automorphisms of $V^{\prime}(\zeta, x)$ which preserve the restriction of the form $f$ to $V^{\prime}(\zeta, x)$. (This restriction is clearly nondegenerate.) Thus in all cases $G(\zeta, x)$ is a classical group of the $j$ th series. If $L(\zeta, x)$ is the Lie algebra of $G(\zeta, x)$ then $x_{\zeta}^{\prime} \in L(\zeta, x)$. Finally let $C(\zeta, x)$ be the centralizer of $x_{\zeta}^{\prime}$ in $G(\zeta, x)$.

Since each of the subspaces $V^{\prime}(\zeta, x)$ is invariant under $C_{G}(x)$, we have

$$
C_{G}(x)=\prod_{\zeta \in \Omega_{j}} C(\zeta, x)
$$

THEOREM 1. Let $G$ be a classical group of the jth series, L its Lie algebra, and $x \in L$ a semisimple element. Then each $C(\zeta, x)$ in $(2.2)$ is a classical group of kth series where $k$ depends only on $j$ and $\zeta$.

For the proof of this theorem we refer to [4].

The values of $k$ are given by Table III (at present, ignore the third column). 


\begin{tabular}{cllc}
$j$ & $\zeta$ & $\lambda$ & $k$ \\
\hline 1 & all $\zeta$ & all $\lambda$ & 1 \\
\hline 2,3 & $\zeta=\bar{\zeta}$ & $\lambda=\bar{\lambda}$ & $k=j$ \\
& $\zeta \neq \bar{\zeta}$ & $\lambda \neq \bar{\lambda}$ & 1 \\
\hline 4 & $\zeta=-\bar{\zeta}$ & $|\lambda|=1$ & 4 \\
& $\zeta \neq-\bar{\zeta}$ & $|\lambda| \neq 1$ & 1 \\
\hline $5 \leqslant j \leqslant 10$ & $\zeta=0$ & $\lambda= \pm 1$ & $k=j$ \\
\hline 5,8 & $\zeta \neq 0$ & $\lambda \neq \pm 1$ & 1 \\
\hline $6,7,9,10$ & $\zeta \neq \pm \bar{\zeta}$ & $\lambda \neq \bar{\lambda} \neq \lambda^{-1}$ & 1 \\
& $\zeta=-\bar{\zeta} \neq 0$ & $\lambda^{-1}=\bar{\lambda} \neq \pm 1$ & 4 \\
\hline 6,9 & $\zeta=\bar{\zeta} \neq 0$ & $\lambda=\bar{\lambda} \neq \pm 1$ & 2 \\
\hline 7,10 & $\zeta=\bar{\zeta} \neq 0$ & $\lambda=\bar{\lambda} \neq \pm 1$ & 3
\end{tabular}

TABLE III

3. Reduction to the nilpotent case. In this section we reduce the problem of finding the closure of a $G$-orbit in $L$ to the case of nilpotent orbits, i.e., orbits consisting of nilpotent elements.

Every $x \in L$ has a unique Jordan decomposition $x=x_{s}+x_{n}$, where $x_{s} \in L$ is semisimple, $x_{n} \in L$ is nilpotent, and $\left[x_{s}, x_{n}\right]=0$. We refer to $x_{s}$ (resp. $x_{n}$ ) as the semisimple (resp. nilpotent) component of $x$.

THEOREM 2. Let $G$ be a classical group, $L$ its Lie algebra, $x$ and $y$ elements of $L$, and $x=x_{s}+x_{n}$ the Jordan decomposition of $x$. Then $y \in \overline{G \cdot x}$ iff $a \cdot y=x_{s}+z$ for some $a \in G$ and some

$$
z \in \overline{C_{G}\left(x_{s}\right) \cdot x_{n}}
$$

For the proof we refer to [4].

Let $\mathcal{\theta}_{1}$ and $\mathcal{O}_{2}$ be $G$-orbits in $L$. Assume that $\mathcal{\theta}_{1} \subset \bar{\theta}_{2}$, which we shall also write as $\theta_{1} \leqslant \theta_{2}$. By Theorem 2 we can choose $x \in \theta_{2}$ and $y \in \theta_{1}$ such that $y=x_{s}+z$ and $z$ satisfies (3.1). Clearly, $z$ is the nilpotent part of $y, z=y_{n}$. By Theorem 1 we have

$$
C_{G}\left(x_{s}\right)=C\left(\zeta_{1}, x_{s}\right) \times \cdots \times C\left(\zeta_{m}, x_{s}\right)
$$

where $\zeta_{1}, \ldots, \zeta_{m}$ are the distinct eigenvalues of $x_{s}$ belonging to $\Omega_{j}$.

Let $Z_{I}\left(x_{s}\right)$ be the centralizer of $x_{s}$ in $L$, i.e., $Z_{L}\left(x_{s}\right)=\left\{t \in L:\left[t, x_{s}\right]=0\right\}$.

We denote by $L\left(\zeta_{k}, x_{s}\right)$ the Lie algebra of $G\left(\zeta_{k}, x_{s}\right)$, and let $Z\left(\zeta_{k}, x_{s}\right)$ be the centralizer in $L\left(\zeta_{k}, x_{s}\right)$ of the restriction of $x_{s}$ to $V^{\prime}\left(\zeta_{k}, x_{s}\right)$. It is clear that $Z_{L}\left(x_{s}\right)$ is the Lie algebra of $C_{G}\left(x_{s}\right)$, and $Z\left(\zeta_{k}, x_{s}\right)$ is the Lie algebra of $C\left(\zeta_{k}, x_{s}\right)$. 
It follows from (3.2) that

$$
Z_{L}\left(x_{s}\right)=Z\left(\zeta_{1}, x_{s}\right) \oplus \cdots \oplus Z\left(\zeta_{m}, x_{s}\right) .
$$

Since $x_{n}, z \in Z_{L}\left(x_{s}\right)$, we have

$$
x_{n}=x_{n}^{(1)}+\cdots+x_{n}^{(m)}, \quad z=z^{(1)}+\cdots+z^{(m)},
$$

where $x_{n}^{(k)}, z^{(k)} \in Z\left(\zeta_{k}, x_{s}\right)$ for $1 \leqslant k \leqslant m$.

It follows from (3.1) to (3.3) that $z^{(k)} \in \overline{C\left(\zeta_{k}, x_{s}\right) \cdot x_{n}^{(k)}}, 1 \leqslant k \leqslant m$.

Thus the $G$-orbits in $L$ which are contained in the closure of the orbit $G \cdot x$ can be easily found provided we know which orbits of $C\left(\zeta_{k}, x_{s}\right)$ in $Z\left(\zeta_{k}, x_{s}\right)$ are contained in the closure of the orbit $C\left(\zeta_{k}, x_{s}\right) \cdot x_{n}^{(k)}, 1 \leqslant k \leqslant n$.

Since $C\left(\zeta_{k}, x_{s}\right)$ is a classical group and $x_{n}^{(k)} \in Z\left(\zeta_{k}, x_{s}\right)$ is nilpotent, we have indeed reduced the general problem to the nilpotent case.

Note that (by assumption) $G$ belongs to the $j$ th series but the groups $C\left(\zeta_{k}, x_{s}\right)$ may belong to some other series of classical groups. This is the reason why we have to consider simultaneously all classical groups.

4. The cases $j=1,2,3,5,8$. Recall that for two $G$-orbits $\theta_{1}$ and $\vartheta_{2}$ in $L$ we write $\theta_{1} \leqslant \theta_{2}$ if $\theta_{1} \subset \bar{\theta}_{2}$.

The cases $j \in\{1,5,8\}$ were treated by M. Gerstenhaber $[5,6]$; his results are more general since he works over an arbitrary algebraically closed field (if $j=5,8$ the characteristic is $\neq 2$ ). The case $j=1$ was also dealt with by J. Dixmier [2]. The cases $j=2,3$ can be treated in the similar way as $j=1$. The cases $j=5,8$ were studied in a more general setting by W. Hesselink [7]. All these authors consider only nilpotent $G$-orbits in $L$, but their results can be easily extended to arbitrary orbits. Finally the cases $j=5,8$ were also considered by the author [3] for arbitrary $G$-orbits in $L$ as well as for conjugacy classes of $G$.

The result for nilpotent $G$-orbits in $L$ can be stated as follows.

THEOREM 3. Let $G$ be a classical group of $j$ th series with $j \in\{1,2,3,5,8\}$, and $L$ its Lie algebra. If $x, y \in L$ are nilpotent then $G \cdot x \leqslant G \cdot y$ holds iff $\operatorname{rank}\left(x^{k}\right) \leqslant \operatorname{rank}\left(y^{k}\right)$ for all $k(\geqslant 0)$.

5. Description of nilpotent orbits. From now on we shall assume that $j \in\{4,6,7,9,10\}$. For a fixed $j$ we consider the triples $(V, f, x)$ where $V$ and $f$ are specified by Table I and $x \in L$, where $L$ is the Lie algebra of the corresponding classical group $G$. An equivalence class of such triples is called a type; see [1] for precise definition. Let $\Delta$ be the type containing the triple $(V, f, x)$. There exists a direct decomposition $V=V_{1} \oplus \cdots \oplus V_{r}$ into $x$-invariant nonzero nondegenerate subspaces which are orthogonal to each other and moreover the summands $V_{1}, \ldots, V_{r}$ cannot be further decomposed in the same sense. Let $x_{k}$ and $f_{k}$ be the restrictions of $x$ and $f$, respectively, to $V_{k}$. If $\Delta_{k}$ is the type containing $\left(V_{k}, f_{k}, x_{k}\right)$ then we shall write $\Delta=\Delta_{1}+\cdots+\Delta_{r}$. We say that $\Delta$ is indecomposable if $\Delta \neq 0$ (i.e., $\operatorname{dim} V \neq 0$ ) and $\Delta$ cannot be written as a sum of two nonzero types. In general, $\Delta$ can be 
expressed as a sum of indecomposable types and as shown in [1] such a decomposition is unique. If $x$ is nilpotent we say that the type $\Delta$ is also nilpotent.

The nilpotent indecomposable types are listed in the second column of Table IV. (At present ignore the last two columns of this table.)

\begin{tabular}{cccc}
$j$ & $\Delta$ & $X$ & $\Phi_{j}$ \\
\hline 4 & $\Delta_{m}^{\varepsilon}(0)$ & $g^{\varepsilon}(m+1)$ & $\Pi$ \\
\hline 6 & $\Delta_{m}^{\varepsilon}(0), m$ even & $g^{\varepsilon}(m+1)$ & $(2 \Lambda, \Pi)$ \\
& $\Delta_{m}(0,0), m$ odd & $2 g(m+1)$ & \\
\hline 7 & $\Delta_{m}(0,0), m$ even & $g(m+1)$ & $(\Pi, \Lambda)$ \\
& $\Delta_{m}^{\varepsilon}(0,0), m$ odd & $g^{\varepsilon}(m+1)$ & \\
\hline 9 & $\Delta_{m}(0,0), m$ even & $2 g(m+1)$ & $(\Pi, 2 \Lambda)$ \\
& $\Delta_{m}^{\varepsilon}(0), m$ odd & $g^{\varepsilon}(m+1)$ & \\
\hline 10 & $\Delta_{m}^{\varepsilon}(0,0), m$ even & $g^{\varepsilon}(m+1)$ & $(\Lambda, \Pi)$ \\
& $\Delta_{m}(0,0), m$ odd & $g(m+1)$ &
\end{tabular}

TABLE IV

In this table $\varepsilon= \pm$ and $m(\geqslant 0)$ is an integer.

We shall now describe representative triples $(V, f, x)$ for each of the types in this table. We warn the reader that our designation of types $\Delta_{m}^{+}$and $\Delta_{m}^{-}$differs from that adopted in [1].

If $j=4$ a representative of $\Delta_{m}^{\varepsilon}(0)$ is given by:

$V$ a complex vector space,

$e_{0}, e_{1}, \ldots, e_{m}$ a basis of $V$,

$$
x\left(e_{k}\right)=e_{k+1} i \quad(i=\sqrt{-1}, 0 \leqslant k<m), \quad x\left(e_{m}\right)=0,
$$

$f$ a hermitian form on $V$,

$f\left(e_{r}, e_{s}\right)=\varepsilon \delta_{r+s, m}\left(\delta_{i j}\right.$ Kronecker symbol).

If $j=6$, a representative of $\Delta_{m}^{\varepsilon}(0), m$ even, say $m=2 m^{\prime}$, is given by:

$V$ a real vector space,

$e_{0}, e_{1}, \ldots, e_{m}$ a basis of $V$,

$$
\begin{aligned}
& x\left(e_{k}\right)=e_{k+1} \quad\left(0 \leqslant k<m^{\prime}\right), \\
& x\left(e_{k}\right)=-e_{k+1} \quad\left(m^{\prime} \leqslant k<m\right), x\left(e_{m}\right)=0,
\end{aligned}
$$

$f$ a symmetric bilinear form on $V$,

$f\left(e_{r}, e_{s}\right)=\varepsilon \delta_{r+s, m}$.

If $j=7$ a representative of $\Delta_{m}^{\varepsilon}(0,0), m$ odd, is given by:

$V$ a (right) quaternionic vector space,

$e_{0}, e_{1}, \ldots, e_{m}$ a basis of $V$,

$x$ given by (5.1),

$f$ a skew-hermitian form on $V$,

$f\left(e_{r}, e_{s}\right)=-i \varepsilon \delta_{r+s, m}$. 
If $j=9$ a representative of $\Delta_{m}^{\varepsilon}(0), m$ odd, say $m=2 m^{\prime}-1$, is given by:

$V$ a real vector space,

$e_{0}, e_{1}, \ldots, e_{m}$ a basis of $V$,

$x$ given by (5.2),

$f$ a skew-symmetric form on $V$,

$f\left(e_{r}, e_{s}\right)=\varepsilon \delta_{r+s, m}$ for $r<s$.

If $j=10$ then a representative of $\Delta_{m}^{\varepsilon}(0,0), m$ even, is given by:

$V$ a (right) quaternionic vector space,

$e_{0}, e_{1}, \ldots, e_{m}$ a basis of $V$,

$x$ given by (5.1),

$f$ a hermitian form on $V$,

$f\left(e_{r}, e_{s}\right)=\varepsilon \delta_{r+s, m}$.

The nilpotent types $\Delta_{m}^{+}$and $\Delta_{m}^{-}$can be distinguished by a simple inequality. Indeed, if $(V, f, x)$ is one of the representatives listed above then for all $v \in V$ we have

$$
\begin{aligned}
(-i)^{m} \varepsilon f\left(v, x^{m}(v)\right) \geqslant 0 . & \text { if } j=4, \\
i^{m} \varepsilon f\left(v, x^{m}(v)\right) \geqslant 0 & \text { if } j=6,10, \\
i^{m-1} \varepsilon f\left(v, x^{m}(v)\right) \geqslant 0 & \text { if } j=7,9 .
\end{aligned}
$$

If $j=6$ (resp. 9) a representative of $\Delta_{m}(0,0), m$ odd (resp. even) is given by: $V$ a real vector space,

$e_{0}, \ldots, e_{m}, e_{0}^{\prime}, \ldots, e_{m}^{\prime}$ a basis of $V$,

$$
\begin{array}{lll}
x\left(e_{k}\right)=e_{k+1} & (0 \leqslant k<m), & x\left(e_{m}\right)=0, \\
x\left(e_{k}^{\prime}\right)=-e_{k+1}^{\prime} & (0 \leqslant k<m), & x\left(e_{m}^{\prime}\right)=0,
\end{array}
$$

$f$ is a symmetric (resp. skew-symmetric) form on $V$,

$$
f\left(e_{r}, e_{s}\right)=f\left(e_{r}^{\prime}, e_{s}^{\prime}\right)=0, f\left(e_{r}, e_{s}^{\prime}\right)=\delta_{r+s, m} .
$$

If $j=7$ then a representative of $\Delta_{m}(0,0)$ with $m$ even has the same description as the representative of $\Delta_{m}^{\varepsilon}(0,0)$ with $m$ odd. (The choice of $\varepsilon= \pm$ is not important since both represent the same type.) Similarly, if $j=10$ then a representative of $\Delta_{m}(0,0)$ with $m$ odd has the same description as the representative of $\Delta_{m}^{\varepsilon}(0,0)$ with $m$ even.

6. Chromosomes. In order to state our results about closures of nilpotent orbits we need certain combinatorial gadgets which we call chromosomes. Roughly speaking they are Young diagrams some of whose rows are filled with alternating + and - signs. A precise definition is given below.

A string (nonpolarized) $S$ is a directed graph isomorphic to

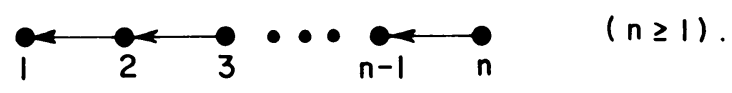


The vertex 1 is the head and the vertex $n$ the tail of this string; $n$ is its rank. If we assign alternating + and - signs to the vertices of this string we obtain a polarized string. A polarized string is positive or negative depending on whether its tail carries a + or - sign. The signature of a polarized string $S$ is the ordered pair

$$
\operatorname{sig} S=\left(r^{+}, r^{-}\right)
$$

where $r^{+}$and $r^{-}$are the numbers of vertices of $S$ with label + and - , respectively. If $S$ is nonpolarized we define its signature by (6.1) where now $r^{+}=r^{-}=\frac{1}{2} n, n$ being the rank of $S$.

A gene is an isomorphism class of strings; it may be polarized or nonpolarized. There are precisely three genes of rank $n$ for each positive integer $n$ : the nonpolarized gene of rank $n$, the positive gene of rank $n$, and the negative gene of rank $n$. We shall denote these three genes in that order by $g(n), g^{+}(n)$, and $g^{-}(n)$.

Let $\Sigma$ be the free abelian group having the set of genes as its basis; the elements of $\Sigma$ will be written as formal finite integral linear combinations of genes. A chromosome is an element of $\Sigma$ which is a nonnegative integral linear combination of genes. The set of all chromosomes will be denoted by $\Sigma^{+}$; it is clearly a free commutative monoid on the set of genes. If $X=Y+Z$ where $Y$ and $Z$ are chromosomes then we say that $X$ contains $Y$, and we write $X \supset Y$ or $Y \subset X$. If $g$ is a gene and $m(\geqslant 0)$ integer such that $X \supset m g$ and $X \not \supset(m+1) g$ then we say that $m$ is the multiplicity of $g$ in the chromosome $X$. We say that $g$ occurs in $X$ if its multiplicity $m$ in $X$ is not zero. The signature of a chromosome $X$ is by definition the sum of the signatures of its genes (taking into account the multiplicities). Similarly, one defines the rank of $X$.

A chromosome is called even (resp. odd, polarized, nonpolarized) if all its constituent genes have even rank (resp. have odd rank, are polarized, are nonpolarized). Every chromosome $X$ admits a unique decomposition $X=e(X)+o(X)$ where $e(X)$ is an even and $o(X)$ an odd chromosome.

A variety is a submonoid of $\Sigma^{+}$. By $\Pi$ (resp. $\Lambda$ ) we denote the variety of polarized (resp. nonpolarized) chromosomes. If $\Phi$ is a variety, so is $k \Phi=\{k X$ : $X \in \Phi\}$, for every nonnegative integer $k$. If $\Phi$ is a variety and $n(\geqslant 0)$ an integer then $\Phi(n)$ denotes the set of all $X \in \Phi$ having rank $n$. If $\Phi$ and $\Psi$ are varieties then $(\Phi, \Psi)$ denotes the variety consisting of all chromosomes $X$ such that $e(X) \in \Phi$ and $o(X) \in \Psi$.

We shall need in the sequel the following five varieties:

$$
\Pi, \quad(\Lambda, \Pi), \quad(\Pi, \Lambda), \quad(2 \Lambda, \Pi), \quad(\Pi, 2 \Lambda) .
$$

If $g$ is a gene of rank $n(>1)$ we define $g^{\prime}$ as follows

$$
\begin{aligned}
g^{\prime} & =g(n-1) & & \text { if } g=g(n), \\
& =g^{+}(n-1) & & \text { if } g=g^{+}(n), \\
& =g^{-}(n-1) & & \text { if } g=g^{-}(n) .
\end{aligned}
$$

If $g$ is a gene of rank 1 we set $g^{\prime}=0$. If $X$ is a chromosome then we define $X^{\prime}$ by applying this "prime" operation to each of its constituent genes. Now we define inductively the chromosome $X^{(k)}, k \geqslant 0$, as follows:

$$
X^{(0)}=X, \quad X^{(k+1)}=\left(X^{(k)}\right)^{\prime} \quad \text { for } k \geqslant 0 .
$$


For instance, if $X=2 g(3)+g^{-}(2)+g(2)+g^{+}(4)$ then

$$
\begin{gathered}
X^{\prime}=X^{(1)}=2 g(2)+g^{-}(1)+g(1)+g^{+}(3), \\
X^{(2)}=2 g(1)+g^{+}(2), \quad X^{(3)}=g^{+}(1), \quad X^{(k)}=0 \quad \text { for } k \geqslant 4 .
\end{gathered}
$$

For ordered pairs of real numbers, the inequality $(p, q) \leqslant(r, s)$ will stand for $p \leqslant r$ and $q \leqslant s$. We say that a chromosome $X$ dominates a chromosome $Y$, and we write $X \geqslant Y$ or $Y \leqslant X$, if

$$
\operatorname{sig} X^{(k)} \geqslant \operatorname{sig} Y^{(k)}, \quad \forall k .
$$

It is easy to check that in each of the varieties (6.2) the dominance relation is a partial order.

7. Statement of the main result. To each indecomposable nilpotent type $\Delta$ of the $j$ th series we associate a chromosome $X=X(\Delta)$ belonging to a variety $\Phi_{j}$. The chromosome $X$ and the variety $\Phi_{j}$ are specified in the last two columns of Table IV.

More generally if $\Delta$ is a nilpotent type of the $j$ th series and $\Delta=\Delta_{1}+\cdots+\Delta_{r}$ is its decomposition into indecomposable types then we define $X(\Delta)=X\left(\Delta_{1}\right)$ $+\cdots+X\left(\Delta_{r}\right)$.

Clearly the map $\Delta \mapsto X(\Delta)$ from the set of nilpotent types of the $j$ th series to $\Phi_{j}$ is a bijection.

Now let $V, \mathbf{F}, f$ and $G$ be as in $\S 1, L$ the Lie algebra of $G$ and $n=\operatorname{dim}_{\mathbf{F}} V$. Since $j \in\{4,6,7,9,10\}$, the signature $\operatorname{sig}(f)$ was defined in $\S 1$. When $j=7$ or 9 we have $\operatorname{sig}(f)=(n / 2, n / 2)$.

Let $\theta$ be a nilpotent $G$-orbit in $L$. If $x, y \in \theta$ then the triples $(V, f, x)$ and $(V, f, y)$ are equivalent and hence we can associate a type $\Delta=\Delta(\theta)$ to the orbit $\theta$. We shall write $X(\theta)$ instead of $X(\Delta(\theta))$.

The following lemma is an immediate consequence of our definitions and the description of nilpotent $G$-orbits in $L$ given in $\$ 5$.

Lemma 4. The map $\theta_{\mapsto} X(\vartheta)$ defined above is a bijection from the set of nilpotent $G$-orbits in $L$ to the set of chromosomes $X \in \Phi_{j}$ satisfying the condition $\operatorname{sig} X=\operatorname{sig}(f)$.

EXAMPLE 1. Let $j=10$ and $\operatorname{sig}(f)=(2,2)$. Then there are six nilpotent orbits in $L$; their types are

$$
\begin{gathered}
2 \Delta_{0}^{+}(0,0)+2 \Delta_{0}^{-}(0,0), \quad \Delta_{0}^{+}(0,0)+\Delta_{0}^{-}(0,0)+\Delta_{1}(0,0), \quad 2 \Delta_{1}(0,0), \\
\Delta_{0}^{+}(0,0)+\Delta_{2}^{-}(0,0), \quad \Delta_{0}^{-}(0,0)+\Delta_{2}^{+}(0,0), \quad \Delta_{3}(0,0) .
\end{gathered}
$$

These types are also listed in [1, p. 351] but the superscripts \pm do not coincide due to the different definition which we have introduced.

The chromosomes in $(\Lambda, \Pi)$ corresponding to these types are $2 g^{+}(1)+2 g^{-}(1)$, $g^{+}(1)+g^{-}(1)+g(2), 2 g(2), g^{+}(1)+g^{-}(3), g^{-}(1)+g^{+}(3)$, and $g(4)$, respectively.

We can now state our main result. Recall that we assume that $G$ is a classical group of the $j$ th series with $j \in\{4,6,7,9,10\}$.

THEOREM 5. If $\vartheta_{1}$ and $\theta_{2}$ are nilpotent orbits of $G$ in its Lie algebra $L$ then $\theta_{1} \subset \bar{\theta}_{2}$ holds iff $X\left(\theta_{1}\right) \leqslant X\left(\theta_{2}\right)$. 
The necessity of the condition $X\left(\theta_{1}\right) \leqslant X\left(\theta_{2}\right)$ was proved in [4]. Our main goal in the remaining part of this paper is to prove the sufficiency of this condition.

EXAMPLE 2. As we saw in Example 1, the group $S p(2,2)$ has 6 nilpotent orbits in its Lie algebra. The partially ordered set of these orbits (with respect to the ordering $\leqslant$ ) is represented by the diagram

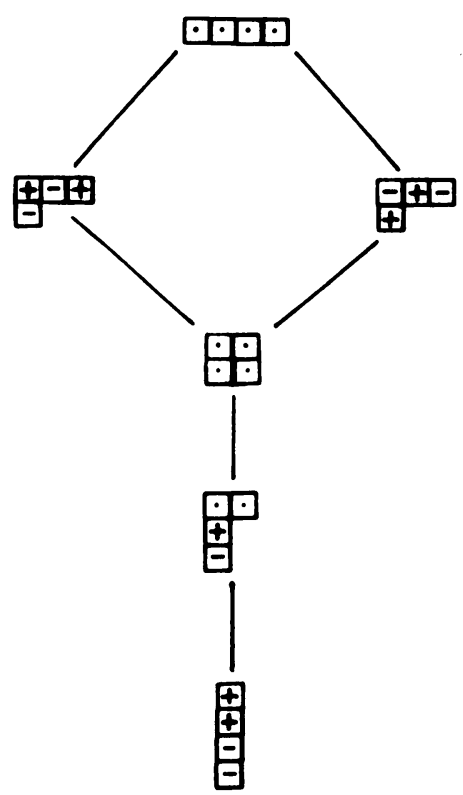

where the orbits are represented by their chromosomes and the rows of dots represent nonpolarized genes. Of course the bottom chromosome $2 g^{+}(1)+2 g^{-}(1)$ represents the trivial orbit consisting of the zero element only.

8. Mutations. The proof of the sufficiency part of Theorem 5 is based on some combinatorial results which we state in this section. The proofs of these results are postponed until the end of the paper.

For each variety $\Phi$ in (6.2) we shall define some transformations of chromosomes which we call $\Phi$-mutations. A chromsome $X \in \Phi$ is transformed by a $\Phi$-mutation into another chromosome $Y \in \Phi$ such that $X \leqslant Y, X \neq Y$, and $\operatorname{sig} X=\operatorname{sig} Y$. We shall indicate such a mutation symbolically by $X \rightarrow Y$. We warn the reader that we may have two $\Phi$-mutations $X \rightarrow Y$ and $X \rightarrow Z$ with $Y \neq Z$ (see the example below).

If $X \rightarrow Y$ is a $\Phi$-mutation and $Z \in \Phi$ then also $X+Z \rightarrow Y+Z$ is a $\Phi$-mutation. Conversely, if $X, Y, Z \in \Phi$ and $X+Z \rightarrow Y+Z$ is a $\Phi$-mutation then also $X \rightarrow Y$ is a $\Phi$-mutation. Hence it suffices to list only the $\Phi$-mutations $X \rightarrow Y$ such that $X$ and $Y$ are disjoint, i.e., $X$ and $Y$ have no genes in common. Such $\Phi$-mutations we call primitive.

The primitive $\Pi$-mutations are the following:

$$
g^{\varepsilon}(m)+g^{-\varepsilon}(n) \rightarrow g^{-\varepsilon}(m-1)+g^{\varepsilon}(n+1), \quad m \leqslant n ;
$$




$$
\begin{array}{cc}
g^{\varepsilon}(m)+g^{\varepsilon}(n) \rightarrow g^{\varepsilon}(m-2)+g^{\varepsilon}(n+2), & 1<m \leqslant n ; \\
g_{\varepsilon}(m)+g_{-\varepsilon}(n) \rightarrow g_{-\varepsilon}(m-1)+g_{\varepsilon}(n+1), & m \leqslant n ;
\end{array}
$$

where $g_{\varepsilon}(n)=g^{(-1)^{n-1} \varepsilon}(n), \varepsilon= \pm$.

EXAMPLE 3. If $X=g^{+}(5)+g^{+}(4)+g^{-(1)}$ and $X \rightarrow Y$ is a $\Pi$-mutation then $Y$ is one of the chormosomes $Y_{1}=g^{-}(6)+g^{+}(4), Y_{2}=g^{+}(5)+g^{-}(5), Y_{3}=g^{+}(6)+$ $g^{+}(3)+g^{-}(1)$, or $Y_{4}=g^{+}(6)+g^{+}(4)$. The mutations $X \rightarrow Y_{1}$ and $X \rightarrow Y_{2}$ are of type (8.1) while the mutations $X \rightarrow Y_{3}$ and $X \rightarrow Y_{4}$ are of type (8.3). (Since $X$ has three genes, none of these mutations is primitive.)

If $\Phi=(\Lambda, \Pi)$ or $(\Pi, \Lambda)$ then the primitive $\Phi$-mutations are the following:

$$
\begin{array}{cc}
g(m)+g(n) \rightarrow g^{\varepsilon}(m-1)+g^{-\varepsilon}(n+1), & m \leqslant n ; \\
g(m)+g^{\varepsilon}(n) \rightarrow g^{\varepsilon}(m-1)+g(n+1), & m<n ; \\
g^{\varepsilon}(m)+g(n) \rightarrow g(m-1)+g^{\varepsilon}(n+1), & m<n ; \\
g^{\varepsilon}(m)+g^{-\varepsilon}(n) \rightarrow g(m-1)+g(n+1), & m \leqslant n ; \\
g^{\varepsilon}(m)+g^{\varepsilon}(n) \rightarrow g^{\varepsilon}(m-2)+g^{\varepsilon}(n+2), & 1<m \leqslant n ;
\end{array}
$$

where $\varepsilon= \pm$. It should be understood that if, say, $\Phi=(\Lambda, \Pi)$ then in (8.4) $m$ and $n$ are even positive integers, etc.

Finally if $\Phi=(2 \Lambda, \Pi)$ or $(\Pi, 2 \Lambda)$ then the primitive $\Phi$-mutations are the following:

$$
\begin{aligned}
& 2 g(m) \rightarrow g^{\varepsilon}(m-1)+g^{-\varepsilon}(m+1) \\
& g^{\varepsilon}(m)+g^{\varepsilon^{\prime}}(n) \rightarrow g^{\varepsilon}(m-2)+g^{\varepsilon^{\prime}}(n+2), \quad 1<m \leqslant n \\
& g^{\varepsilon}(m)+g^{+}(n)+g^{-}(n) \rightarrow g^{\varepsilon}(m-2)+2 g(n+1), \quad 1<m \leqslant n \\
& g^{+}(m)+g^{-}(m)+g^{\varepsilon}(n) \rightarrow 2 g(m-1)+g^{\varepsilon}(n+2), \quad m \leqslant n \\
& g^{+}(m)+g^{-}(m)+g^{+}(n)+g^{-}(n) \rightarrow 2 g(m-1)+2 g(n+1), \quad m \leqslant n \\
& 2 g^{\varepsilon}(m)+2 g^{-\varepsilon}(n) \rightarrow 2 g(m-1)+2 g(n+1), \quad m \leqslant n \\
& g^{\varepsilon}(m)+g^{-\varepsilon}(n) \rightarrow g^{-\varepsilon}(m-2)+g^{\varepsilon}(n+2), \quad 1<m \leqslant n \\
& 2 g^{\varepsilon}(m)+g^{-\varepsilon}(n) \rightarrow 2 g(m-1)+g^{\varepsilon}(n+2), \quad m \leqslant n \\
& g^{\varepsilon}(m)+2 g^{-\varepsilon}(n) \rightarrow g^{-\varepsilon}(m-2)+2 g(n+1), \quad 1<m \leqslant n
\end{aligned}
$$

where $\varepsilon, \varepsilon^{\prime}= \pm$. It should be also understood that $g(0)$ and $g^{\varepsilon}(0), \varepsilon= \pm$, are zero chromosomes.

This completes the definition of $\Phi$-mutations when $\Phi$ is one of the varieties (6.2).

We say that there are enough $\Phi$-mutations if given $X, Y \in \Phi$, such that $X \leqslant Y$ and $\operatorname{sig} X=\operatorname{sig} Y$, there exists a finite chain of $\Phi$-mutations

$$
X_{0} \rightarrow X_{1} \rightarrow X_{2} \rightarrow \cdots \rightarrow X_{m} \quad(m \geqslant 0)
$$

with $X_{0}=X$ and $X_{m}=Y$.

The combinatorial result which we need is the following.

THEOREM 6. If $\Phi$ is one of the varieties (6.2) then there are enough $\Phi$-mutations. 
The proof of this theorem is given in $\S \S 14-17$. It is much harder than the proof of the analogous results for the varieties $\Lambda,(2 \Lambda, \Lambda)$, and $(\Lambda, 2 \Lambda)$ which are given in [3] and [7].

9. Sufficiency part of Theorem 5. Recall that $G$ is a classical group of the $j$ th series defined by a pair $(V, f)$ and $j \in\{4,6,7,9,10\}$. Let $\theta_{1}$ and $\theta_{2}$ be two nilpotent orbits of $G$ in its Lie algebra $L$. Let $X_{1}=X\left(\theta_{1}\right)$ and $X_{2}=X\left(\theta_{2}\right)$ be the corresponding chromosomes in $\Phi_{j}$. By hypothesis we have $X_{1} \leqslant X_{2}$. We also know that $\operatorname{sig} X_{1}=\operatorname{sig} X_{2}=\operatorname{sig}(f)$. We have to show that $\mathcal{\theta}_{1} \leqslant \theta_{2}$, i.e., $\mathcal{O}_{1} \subset \bar{\theta}_{2}$. By Theorem 6 it suffices to consider the case when

$$
X_{1} \rightarrow X_{2}
$$

is a $\Phi_{j}$-mutation. Without any loss of generality we may (and we will) assume that this mutation is primitive.

In order to prove that $\theta_{1} \leqslant \theta_{2}$ we shall construct a linear one-parameter family

$$
x(t)=x_{0}+t x_{1}
$$

in $L$ such that $x_{0} \in \mathcal{O}_{1}$ and $x(t) \in \mathcal{O}_{2}$ for $t>0$. (Only in one case we shall use a quadratic family.) Indeed the existence of such a family implies that $x_{0} \in \overline{\mathcal{O}}_{2}$, and so $\theta_{1} \subset \bar{\theta}_{2}$.

In order to verify that, say, $x_{0} \in \mathcal{O}_{1}$ it suffices to show that $\left(V, f, x_{0}\right) \in \Delta\left(\theta_{1}\right)$. For this purpose we decompose $V$ into an orthogonal direct sum of $x_{0}$-invariant subspaces $V_{1}, \ldots, V_{k}$ so that the corresponding types $\Delta_{1}, \ldots, \Delta_{k}$ are indecomposable. We identify these indecomposable types by the criteria of $\$ 5$ and verify that $\Delta\left(\mathbb{O}_{1}\right)=\Delta_{1}+\cdots+\Delta_{k}$. The same method is used to prove that $x(t) \in \mathbb{\theta}_{2}$ for $t>0$.

We shall distinguish several cases according to the value of $j$.

10. The case $j=4$. We have $\Phi_{4}=\Pi$ and the primitive $\Pi$-mutations are defined by (8.1)-(8.3). These mutations can also be written as

$$
\begin{aligned}
& g^{\varepsilon}(m+1)+g^{\varepsilon^{\prime}}(n+1) \rightarrow g^{\varepsilon^{\prime}}(m)+g^{\varepsilon}(n+2), \quad n-m=2 k+1 \\
& g^{\varepsilon}(m+1)+g^{\varepsilon}(n+1) \rightarrow g^{\varepsilon}(m-1)+g^{\varepsilon}(n+3), \quad m \leqslant n \\
& g^{-\varepsilon}(m+1)+g^{\varepsilon}(n+1) \rightarrow g^{-\varepsilon^{\prime}}(m)+g^{\varepsilon^{\prime}}(n+2), \quad n-m=2 k
\end{aligned}
$$

where $k(\geqslant 0)$ is an integer and $\varepsilon, \varepsilon^{\prime}= \pm$.

Indeed, (8.2) and (10.2) list the same mutations. Mutations (8.1) for $n-m$ even are the same as (10.3) for $\varepsilon^{\prime}=\varepsilon$. Mutations (8.1) for $n-m$ odd are the same as (10.1) for $\varepsilon^{\prime}=-\varepsilon$. Mutations (8.3) for $n-m$ even are the same as (10.3) for $\varepsilon^{\prime}=\varepsilon$. Finally, mutations (8.3) for $n-m$ odd are the same as (10.1) for $\varepsilon^{\prime}=\varepsilon$.

Assume first that the mutation (9.1) is of the form (10.1). We can choose a basis $e_{0}, \ldots, e_{m}, e_{0}^{\prime}, \ldots, e_{n}^{\prime}$ of $V$ such that

$$
f\left(e_{r}, e_{s}^{\prime}\right)=0, f\left(e_{r}, e_{s}\right)=\varepsilon \boldsymbol{\delta}_{r+s, m}, f\left(e_{r}^{\prime}, e_{s}^{\prime}\right)=\varepsilon^{\prime} \delta_{r+s, n}
$$

holds for all relevant $r, s$. We define linear maps $x_{0}$ and $x_{1}$ on $V$ by specifying their effect on basis elements:

$$
\begin{array}{ll}
x_{0} e_{r}=i e_{r+1} & (0 \leqslant r<m), \quad x_{0} e_{m}=0, \\
x_{0} e_{r}^{\prime}=i e_{r+1}^{\prime} & (0 \leqslant r<n), \quad x_{0} e_{n}^{\prime}=0,
\end{array}
$$




$$
\begin{aligned}
& x_{1} e_{r}=0 \quad(0 \leqslant r<m), \quad x_{1} e_{m}=i e_{n-k}^{\prime}, \\
& x_{1} e_{r}^{\prime}=0 \quad \text { for } r \neq k, \quad x_{1} e_{k}^{\prime}=i \varepsilon \varepsilon^{\prime} e_{0} .
\end{aligned}
$$

In view of (5.1) and the definition of $x_{0}$ it is clear that $x_{0} \in \vartheta_{1}$. It is easy to check that $x_{1} \in L$ and so $x(t) \in L$ for all real $t$. We have

$$
\begin{aligned}
& x(t)^{k+1} e_{0}^{\prime}=x(t) i^{k} e_{k}^{\prime}=i^{k+1}\left(e_{k+1}^{\prime}+\varepsilon \varepsilon^{\prime} t e_{0}\right), \\
& x(t)^{k+m+1} e_{0}^{\prime}=i^{k+m+1}\left(e_{k+m+1}^{\prime}+\varepsilon \varepsilon^{\prime} t e_{m}\right)=i^{n-k}\left(e_{n-k}^{\prime}+\varepsilon \varepsilon^{\prime} t e_{m}\right), \\
& x(t)^{n} e_{0}^{\prime}=i^{n-k} x(t)^{k}\left(e_{n-k}^{\prime}+\varepsilon \varepsilon^{\prime} t e_{m}\right)=i^{n}\left(e_{n}^{\prime}+\varepsilon \varepsilon^{\prime} t^{2} e_{n-1}^{\prime}\right), \\
& x(t)^{n+1} e_{0}^{\prime}=i^{n+1} \varepsilon \varepsilon^{\prime} t^{2} e_{n}^{\prime}, \quad x(t)^{n+2} e_{0}^{\prime}=0, \\
& x(t)^{m-1}\left(e_{1}-t e_{k+1}^{\prime}\right)=i^{m-1}\left(e_{m}-t e_{k+m}^{\prime}\right)=i^{m-1}\left(e_{m}-t e_{n-k-1}^{\prime}\right), \\
& x(t)^{m}\left(e_{1}-t e_{k+1}^{\prime}\right)=0 .
\end{aligned}
$$

We now assume that $t \neq 0$. Let $V_{1}$ and $V_{2}$ be the $x(t)$-invariant subspaces generated by the vectors $e_{0}^{\prime}$ and $e=e_{1}-t e_{k+1}^{\prime}$, respectively. It is clear that $V=V_{1} \oplus V_{2}$, $\operatorname{dim} V_{1}=n+2, \operatorname{dim} V_{2}=m$. Note that the vectors $e_{r}-t e_{r+k}^{\prime}(1 \leqslant r \leqslant m)$ form a basis of $V_{2}$ and are orthogonal to $e_{0}^{\prime}$. Hence $V_{1} \cap V_{2}^{\perp}$ is an $x(t)$-invariant subspace of $V_{1}$ containing $e_{0}^{\prime}$. It follows from the definition of $V_{1}$ that $V_{1} \cap V_{2}^{\perp}=V_{1}$, i.e., $V_{1}=V_{2}^{\perp}$. Let $\Delta_{1}$ and $\Delta_{2}$ be the types which correspond to $V_{1}$ and $V_{2}$, respectively. Since

$$
\varepsilon(-i)^{n+1} f\left(e_{0}^{\prime}, x(t)^{n+1} e_{0}^{\prime}\right)=t^{2}=\varepsilon^{\prime}(-i)^{m-1} f\left(e, x(t)^{m-1} e\right),
$$

it follows from (5.3) that

$$
\Delta_{1}=\Delta_{n+1}^{\varepsilon}(0), \quad \Delta_{2}=\Delta_{m-1}^{\varepsilon^{\prime}}(0) .
$$

Hence indeed $\Delta_{1}+\Delta_{2}=\Delta\left(\vartheta_{2}\right)$, and so $x(t) \in \vartheta_{2}$ for $t \neq 0$.

Next assume that the mutation (9.1) is of the form (10.2). Then we can choose a basis $e_{0}, \ldots, e_{m}, e_{0}^{\prime}, \ldots, e_{n}^{\prime}$ of $V$ such that (10.4) holds with $\varepsilon^{\prime}=\varepsilon$. We define $x(t)$ by

$$
\begin{aligned}
& x(t) e_{0}=i\left(e_{1}+t e_{0}^{\prime}\right), \quad x(t) e_{r}=i e_{r+1} \quad(0<r<m), \quad x(t) e_{m}=0, \\
& x(t) e_{r}^{\prime}=i e_{r+1}^{\prime} \quad(0 \leqslant r<n), \quad x(t) e_{n}^{\prime}=i t e_{m} .
\end{aligned}
$$

As in the previous case we have $x_{0} \in \vartheta_{1}$ and $x_{1} \in L$. We have

$$
\begin{aligned}
& x(t)^{m} e_{0}=i x(t)^{m-1}\left(e_{1}+t e_{0}^{\prime}\right)=i^{m}\left(e_{m}+t e_{m-1}^{\prime}\right), \\
& x(t)^{n+2} e_{0}=i^{m} x(t)^{n-m+2} t e_{m-1}^{\prime}=i^{n+1} t x(t) e_{n}^{\prime}=i^{n+2} t^{2} e_{m}, \\
& x(t)^{n+3} e_{0}=0 .
\end{aligned}
$$

If $m \neq 1$ we have

$$
x(t)^{m-2}\left(t e_{1}-e_{n-m+2}^{\prime}\right)=i^{m-2}\left(t e_{m-1}-e_{n}^{\prime}\right), x(t)^{m-1}\left(t e_{1}-e_{n-m+2}^{\prime}\right)=0 .
$$

Let $t \neq 0$ and let $V_{1}$ be the $x(t)$-invariant subspace generated by $e_{0}$. Clearly, $\operatorname{dim} V_{1}=n+3$ and so if $m=1$ we have $V_{1}=V$. When $m \neq 1$ let $V_{2}$ be the $x(t)$-invariant subspace generated by the vector $e=t e_{1}-e_{n-m+2}^{\prime}$. Clearly, $\operatorname{dim} V_{2}=m-1$ and $V=V_{1} \oplus V_{2}$. The vectors $t e_{r}-e_{n-m+r+1}^{\prime}(1 \leqslant r \leqslant m-1)$ form a basis of $V_{2}$ and are orthogonal to $e_{0}$. As in the previous case this implies that 
$V_{1}=V_{2}^{\perp}$. Let $\Delta_{1}$ be the type associated with $V_{1}$ and if $m \neq 1$ let $\Delta_{2}$ be the type associated with $V_{2}$. Since

$$
\varepsilon(-i)^{n+2} f\left(e_{0}, x(t)^{n+2} e_{0}\right)=t^{2},
$$

and if $m \neq 1$

$$
\varepsilon(-i)^{m-2} f\left(e, x(t)^{m-2} e\right)=t^{2},
$$

we have $\Delta_{1}=\Delta_{n+2}^{\varepsilon}(0), \Delta_{2}=\Delta_{m-2}^{\varepsilon}(0)(m \neq 1)$. Thus $\Delta_{1}=\Delta\left(\theta_{2}\right)$ if $m=1$ and $\Delta_{1}+\Delta_{2}=\Delta\left(\theta_{2}\right)$ if $m \neq 1$. Therefore $x(t) \in \theta_{2}$ for $t \neq 0$.

Finally assume that the mutation (9.1) is of the form (10.3). Then we can choose a basis $e_{0}, \ldots, e_{m+k}, e_{0}^{\prime}, \ldots, e_{m+k}^{\prime}$ of $V$ such that

$$
f\left(e_{r}, e_{s}\right)=f\left(e_{r}^{\prime}, e_{s}^{\prime}\right)=0, \quad f\left(e_{r}, e_{s}^{\prime}\right)=\delta_{r+s, m+k}
$$

holds for all $r, s$. We define linear operators $x_{0}$ and $x_{1}$ on $V$ by

$$
\begin{aligned}
& x_{0} e_{r}=i e_{r+1} \quad \text { for } r \neq k-1, m+k, \\
& x_{0} e_{k-1}=i\left(e_{k}+\varepsilon e_{0}^{\prime}\right) \quad \text { if } k>0, \\
& x_{0} e_{m+k}=i \varepsilon e_{m+1}^{\prime} \quad\left(e_{m+1}^{\prime}=0 \text { if } k=0\right), \\
& x_{0} e_{r}^{\prime}=i e_{r+1}^{\prime} \quad(0 \leqslant r<m+k), \quad x_{0} e_{m+k}^{\prime}=0 ; \\
& x_{1} e_{r}=0 \quad \text { for } r \neq k, m+k, \\
& x_{1} e_{k}=i \varepsilon^{\prime} e_{0}^{\prime}, \quad x_{1} e_{m+k}=i \varepsilon^{\prime} e_{m}^{\prime}, \\
& x_{1} e_{r}^{\prime}=0 \quad(0 \leqslant r \leqslant m+k) .
\end{aligned}
$$

From the definition of $x_{0}$ and from (10.5) it follows that $f\left(x_{0} e_{r}, e_{s}\right)=0=f\left(e_{r}, x_{0} e_{s}\right)$ for all $r, s$ except (when $\left.k>0\right)$ that

$$
\begin{aligned}
& f\left(x_{0} e_{k-1}, e_{m+k}\right)=-i \varepsilon=f\left(x_{0} e_{m+k}, e_{k-1}\right), \\
& f\left(e_{k-1}, x_{0} e_{m+k}\right)=i \varepsilon=f\left(e_{m+k}, x_{0} e_{k-1}\right) .
\end{aligned}
$$

Hence for all $r, s$ we have $f\left(x_{0} e_{r}, e_{s}\right)+f\left(e_{r}, x_{0} e_{s}\right)=0$.

Since the subspace spanned by $e_{0}^{\prime}, \ldots, e_{m+k}^{\prime}$ is totally isotropic it is clear that $f\left(x_{0} e_{r}^{\prime}, e_{s}^{\prime}\right)+f\left(e_{r}^{\prime}, x_{0} e_{s}^{\prime}\right)=0$ holds for all $r, s$.

We have $f\left(x_{0} e_{r}, e_{s}^{\prime}\right)=0=f\left(e_{r}, x_{0} e_{s}^{\prime}\right)$ for all $r, s$ except that

$$
\begin{array}{ll}
f\left(x_{0} e_{r}, e_{m+k-r-1}^{\prime}\right)=i & (0 \leqslant r<m+k), \\
f\left(e_{r}, x_{0} e_{m+k-r-1}^{\prime}\right)=-i & (0 \leqslant r<m+k) .
\end{array}
$$

Thus for all $r, s$ we have $f\left(x_{0} e_{r}, e_{s}^{\prime}\right)+f\left(e_{r}, x_{0} e_{s}^{\prime}\right)=0$.

Since $f$ is a hermitian form, it follows that $f\left(x_{0} v_{1}, v_{2}\right)+f\left(v_{1}, x_{0} v_{2}\right)=0$ for all $v_{1}, v_{2} \in V$, i.e., $x_{0} \in L$.

It is obvious that $f\left(x_{1} e_{r}, e_{s}^{\prime}\right)+f\left(e_{r}, x_{1} e_{s}^{\prime}\right)=0$ and $f\left(x_{1} e_{r}^{\prime}, e_{s}^{\prime}\right)+f\left(e_{r}^{\prime}, x_{1} e_{s}^{\prime}\right)=0$ for all $r, s$. We have $f\left(x_{1} e_{r}, e_{s}\right)=0=f\left(e_{r}, x_{1} e_{s}\right)$ for all $r, s$ except for

$$
\begin{aligned}
& f\left(x_{1} e_{k}, e_{m+k}\right)=-i \varepsilon^{\prime}=f\left(x_{1} e_{m+k}, e_{k}\right), \\
& f\left(e_{k}, x_{1} e_{m+k}\right)=i \varepsilon^{\prime}=f\left(e_{m+k}, x_{1} e_{k}\right) .
\end{aligned}
$$

Hence $f\left(x_{1} e_{r}, e_{s}\right)+f\left(e_{r}, x_{1} e_{s}\right)=0$ for all $r, s$. Therefore $x_{1} \in L$ and $x(t)=x_{0}+t x_{1} \in L$ for all real $t$. 
We claim that $x_{0} \in \mathcal{O}_{1}$. Let first $k=0$, i.e., $m=n$. For real $\lambda$ let $U_{\lambda}$ be the $x_{0}$-invariant subspace generated by the vector $e_{0}+\lambda e_{0}^{\prime}$. The vectors $e_{r}+\lambda e_{r}^{\prime}$ $(0 \leqslant r \leqslant m)$ form a basis of $U_{\lambda}$. Since

$$
\varepsilon(-i)^{m} f\left(e_{0}+\lambda e_{0}^{\prime}, x_{0}^{m}\left(e_{0}+\lambda e_{0}^{\prime}\right)\right)=2 \varepsilon \lambda,
$$

$U_{\lambda}$ is nondegenerate for $\lambda \neq 0$. (Indeed, if degenerate then $x_{0}^{m}\left(e_{0}+\lambda e_{0}^{\prime}\right)$ would be in its radical.) Since $e_{0}+e_{0}^{\prime}$ is orthogonal to $e_{r}-e_{r}^{\prime}(0 \leqslant r \leqslant m)$, it follows that $V=U_{1} \oplus U_{-1}$ and $U_{1} \perp U_{-1}$. From (10.6) and (5.3) it follows now that $\left(V, f, x_{0}\right) \in \Delta_{m}^{+}(0)+\Delta_{m}^{-}(0)$, and so $x_{0} \in \mathcal{O}_{1}$. Now let $k>0$. We have then

$$
\begin{aligned}
& x_{0}^{n} e_{0}=i^{k} x_{0}^{n-k}\left(e_{k}+\varepsilon e_{0}^{\prime}\right)=i^{m+k} x_{0}^{k}\left(e_{m+k}+\varepsilon e_{m}^{\prime}\right)=2 \varepsilon i^{n} e_{m+k}^{\prime}, \\
& x_{0}^{n+1} e_{0}=0, \quad x_{0}^{m}\left(e_{k}-\varepsilon e_{0}^{\prime}\right)=i^{m}\left(e_{m+k}-\varepsilon e_{m}^{\prime}\right), \\
& x_{0}^{m+1}\left(e_{k}-\varepsilon e_{0}^{\prime}\right)=0 .
\end{aligned}
$$

Let $V_{1}$ and $V_{2}$ be the $x_{0}$-invariant subspaces generated by $e_{0}$ and $e=e_{k}-\varepsilon e_{0}^{\prime}$, respectively. Since $e_{m+k}^{\prime}$ and $e_{k}-\varepsilon e_{0}$ are linearly independent vectors in $\operatorname{Ker} x_{0}$, the above equalities imply that $V=V_{1} \oplus V_{2}, \operatorname{dim} V_{1}=n+1$ and $\operatorname{dim} V_{2}=m+1$. Since the vectors $e_{k+r}-\varepsilon e_{r}^{\prime}(0 \leqslant r \leqslant m)$ form a basis of $V_{2}$ and are orthogonal to $e_{0}$, we have $V_{1} \perp V_{2}$. Since $\varepsilon(-i)^{n} f\left(e_{0}, x_{0}^{n} e_{0}\right)=2=-\varepsilon(-i)^{m} f\left(e, x_{0}^{m} e\right)$, it follows from $\S 5$ that $\left(V, f, x_{0}\right) \in \Delta_{m}^{-\varepsilon}(0)+\Delta_{n}^{\varepsilon}(0)$. Thus again $x_{0} \in \mathcal{O}_{1}$.

For $k>0$ we have

$$
\begin{aligned}
x(t)^{n+1} e_{0} & =i^{k} x(t)^{n-k+1}\left(e_{k}+\varepsilon e_{0}^{\prime}\right)=i^{k+1} x(t)^{n-k}\left(e_{k+1}+\varepsilon^{\prime} t e_{0}^{\prime}+\varepsilon e_{1}^{\prime}\right) \\
& =i^{m+k} x(t)^{k+1}\left(e_{m+k}+\varepsilon^{\prime} t e_{m-1}^{\prime}+\varepsilon e_{m}^{\prime}\right) \\
& =2 i^{m+k+1} x(t)^{k}\left(\varepsilon^{\prime} t e_{m}^{\prime}+\varepsilon e_{m+1}^{\prime}\right)=2 i^{n+1} \varepsilon^{\prime} t e_{m+k}^{\prime},
\end{aligned}
$$

and for $k=0$

$$
x(t)^{n+1} e_{0}=i x(t)^{n}\left(e_{1}+\varepsilon^{\prime} t e_{0}^{\prime}\right)=i^{n} x(t)\left(e_{n}+\varepsilon^{\prime} t e_{n-1}^{\prime}\right)=2 i^{n+1} \varepsilon^{\prime} t e_{n}^{\prime} .
$$

Thus for all $k$ we have

$$
x(t)^{n+1} e_{0}=2 i^{n+1} \varepsilon^{\prime} t e_{m+k}^{\prime}, \quad x(t)^{n+2} e_{0}=0 .
$$

Writing $a=e_{k+1}-\varepsilon^{\prime} t e_{0}^{\prime}-\varepsilon e_{1}^{\prime}$, we have

$$
x(t)^{m-1} a=i^{m-1}\left(e_{m+k}-\varepsilon^{\prime} t e_{m-1}^{\prime}-\varepsilon e_{m}^{\prime}\right), \quad x(t)^{m} a=0 .
$$

Fix $t \neq 0$ and let $W_{1}$ and $W_{2}$ be $x(t)$-invariant subspaces generated by $e_{0}$ and $a$, respectively. Let first $k>0$. Then the vectors $e_{r+k}-\varepsilon^{\prime} t e_{r-1}^{\prime}-\varepsilon e_{r}^{\prime}(1 \leqslant r \leqslant m)$ form a basis of $W_{2}$ and are orthogonal to $e_{0}$. From (10.7) and (10.8) we conclude that $V=W_{1} \oplus W_{2}, \operatorname{dim} W_{1}=n+2, \operatorname{dim} W_{2}=m$, and $W_{1} \perp W_{2}$. Now let $k=0$ and let $W_{3}$ be the $x(t)$-invariant subspace generated by the vector $b=e_{1}-\varepsilon^{\prime} t e_{0}^{\prime}$. Then $x(t)^{m-1} b=i^{m-1}\left(e_{m}-\varepsilon^{\prime} t e_{m-1}^{\prime}\right), \quad x(t)^{m} b=0$. Hence the vectors $e_{r}-\varepsilon^{\prime} t e_{r-1}^{\prime}$ $(1 \leqslant r \leqslant m)$ form a basis of $W_{3}$ and are orthogonal to $e_{0}$. It follows that $V=W_{1} \oplus W_{3}$ and $W_{1} \perp W_{3}$. 
Since

$$
\begin{aligned}
\varepsilon^{\prime}(-i)^{n+1} f\left(e_{0}, x(t)^{n+1} e_{0}\right) & =2 t, \\
-\varepsilon^{\prime}(-i)^{m-1} f\left(a, x(t)^{m-1} a\right) & =2 t, \\
-\varepsilon^{\prime}(-i)^{m-1} f\left(b, x(t)^{m-1} a\right) & =2 t \quad(k=0),
\end{aligned}
$$

it follows that $x(t) \in \theta_{2}$ for $t>0$.

11. The cases $j=7,10$. We shall reduce these cases to the previous case $j=4$. Recall that $\Phi_{7}=(\Pi, \Lambda)$ and $\Phi_{10}=(\Lambda, \Pi)$.

Given $X \in \Pi$ we shall denote by $\sigma(X)$, resp. $\tau(X)$, the chromosome in $(\Pi, \Lambda)$, resp. $(\Lambda, \Pi)$, which is obtained from $X$ by ignoring the polarization of its odd, resp. even, genes. Clearly $\sigma: \Pi \rightarrow(\Pi, \Lambda)$ and $\tau: \Pi \rightarrow(\Lambda, \Pi)$ are morphisms of monoids.

LEMma 7. Every primitive $(\Pi, \Lambda)$-mutation, resp. $(\Lambda, \Pi)$-mutation, is of the form $\sigma(X) \rightarrow \sigma(Y)$, resp. $\tau(X) \rightarrow \tau(Y)$, for a suitable primitive $\Pi$-mutation $X \rightarrow Y$.

ProOF. We have to show that each of the mutations (8.4)-(8.8) can be obtained by applying $\sigma$ or $\tau$ to a primitive $\Pi$-mutation $X \rightarrow Y$. In the case (8.4) we take $X=g^{-\varepsilon}(m)+g^{\varepsilon}(n), \quad Y$ is determined by (8.4). In the case (8.5) we take $X=g^{-\varepsilon}(m)+g^{\varepsilon}(n), \quad Y=g^{\varepsilon}(m-1)+g^{-\varepsilon}(n+1)$. In the case (8.6) we take $X=g^{\varepsilon}(m)+g^{-\varepsilon}(n), \quad Y=g^{-\varepsilon}(m-1)+g^{\varepsilon}(n+1)$. In the case (8.7) we take $Y=g^{-\varepsilon}(m-1)+g^{\varepsilon}(n+1), X$ being already determined by (8.7). The mutations (8.8) are also primitive $\Pi$-mutations.

Let $U$ be a classical group of the fourth series defined by a pair $(W, g)$. Let $W^{\prime}=W \otimes_{\mathbf{C}} \mathbf{H}$ and let $g_{\sigma}$, resp. $g_{\tau}$, be the skew-hermitian, resp. hermitian, form on $W^{\prime}$ whose restriction to $W$ is $-i g$, resp. $g$. Since $g$ is nondegenerate, so are $g_{\sigma}$ and $g_{\tau}$. Let $U_{\sigma}$, resp. $U_{\tau}$, be the classical group of the seventh, resp. tenth, series defined by the pair $\left(W^{\prime}, g_{\sigma}\right)$, resp. $\left(W^{\prime}, g_{\tau}\right)$. Clearly we may view $U$ as a subgroup of $U_{\sigma}$ and $U_{\tau}$. Let $M$ (resp. $M_{\sigma}, M_{\tau}$ ) be the Lie algebra of $U$ (resp. $U_{\sigma}, U_{\tau}$ ). We shall view $M$ as a subalgebra of $M_{\sigma}$ and $M_{\tau}$. Let $x \in M$ be a nilpotent element, $\Delta$ the type determined by $(W, g, x)$ and $X=X(\Delta) \in \Pi$ the corresponding chromosome. Similarly, let $\Delta_{\sigma}$, resp. $\Delta_{\tau}$, be the type determined by $\left(W^{\prime}, g_{\sigma}, x\right)$, resp. $\left(W^{\prime}, g_{\tau}, x\right)$, and let $X_{\sigma}$, resp. $X_{\tau}$, be its chromosome in $(\Pi, \Lambda)$, resp. $(\Lambda, \Pi)$.

LEMma 8. With the above notation we have $X_{\sigma}=\sigma(X)$ and $X_{\tau}=\tau(X)$.

Proof. It suffices to prove this when $\Delta$ is indecomposable, i.e., $X$ is a gene, say $X=g^{\varepsilon}(m+1)$. We may assume (see $\left.\S 5\right)$ that there is a basis $e_{0}, \ldots, e_{m}$ of $W$ such that

$$
g\left(e_{r}, e_{s}\right)=\varepsilon \delta_{r+s, m}
$$

for all $r, s$ and

$$
x e_{r}=e_{r+1} i \quad(0 \leqslant r<m), \quad x e_{m}=0 .
$$

Since $W^{\prime}$ is indecomposable with respect to $x$, we must have $X_{\sigma}=g(m+1)$ for $m$ even and $X_{\tau}=g(m+1)$ for $m$ odd. If $m$ is odd we have $i^{m-1} \varepsilon g_{\sigma}\left(e_{0}, x^{m} e_{0}\right)=$ $-i^{m} \varepsilon g\left(e_{0}, e_{m} i^{m}\right)=1$ by $(11.1),(11.2)$ and the definition of $g_{\sigma}$. By (5.5) we have 
$X_{\sigma}=g^{\varepsilon}(m+1)$ in this case. If $m$ is even then $i^{m} \varepsilon g_{\tau}\left(e_{0}, x^{m} e_{0}\right)=i^{m} \varepsilon g\left(e_{0}, e_{m} i^{m}\right)=1$, and so by (5.4) we have $X_{\tau}=g^{\varepsilon}(m+1)$.

Thus $X_{\sigma}=\sigma(X)$ and $X_{\tau}=\tau(X)$ in all cases.

We now proceed with our proof of the sufficiency part of Theorem 5. We shall consider in detail the case $j=7$. Then, by hypothesis, (9.1) is a primitive $(\Pi, \Lambda)$-mutation. By Lemma 7 there exists a primitive $\Pi$-mutation $Y_{1} \rightarrow Y_{2}$ such that $\sigma\left(Y_{r}\right)=X_{r}(r=1,2)$.

From $\S 5$ it is easy to deduce that there exists a $C$-subspace $V_{0}$ of $V$ such that the canonical map $V_{0} \otimes_{\mathbf{C}} \mathbf{H} \rightarrow V$ is an isomorphism, $f$ takes complex values on $V_{0} \times V_{0}$, and $\operatorname{sig}\left(i f_{0}\right)=\operatorname{sig} Y_{1}=\operatorname{sig} Y_{2}$ where $f_{0}$ is the restriction of $f$ to $V_{0}$. Let $U$ be the classical group of the fourth series defined by the pair $\left(V_{0}, i f_{0}\right)$. Then we have $U_{\sigma}=G$. Let $\Theta_{r}^{\prime}(r=1,2)$ be the nilpotent orbit of $U$ in its Lie algebra $M$ with the associated chromosome $Y_{r}$. If we identify $M_{\sigma}$ with $L$, then Lemma 8 implies that $\theta_{r}^{\prime} \subset \theta_{r}(r=1,2)$. Since $Y_{1} \leqslant Y_{2}$, we have $\theta_{1}^{\prime} \leqslant \theta_{2}^{\prime}$ by the case $j=4$. This clearly implies that $\theta_{1} \leqslant \theta_{2}$.

The proof in the case $j=10$ is similar and we may safely omit the details.

12. The cases $j=6,9$. These two cases will be treated simultaneously. Recall that $\Phi_{6}=(2 \Lambda, \Pi)$ and $\Phi_{9}=(\Pi, 2 \Lambda)$.

If $m(\geqslant 0)$ is an integer we shall write $m^{\prime}=\left[\frac{1}{2}(m+1)\right]$. Thus $m=2 m^{\prime}$ for $m$ even and $m=2 m^{\prime}-1$ for $m$ odd. We have

$$
(-1)^{m-m^{\prime}}= \begin{cases}i^{m} & \text { if } m \text { is even, } \\ i^{m-1} & \text { if } m \text { is odd. }\end{cases}
$$

Assume first that the mutation (9.1) has the form

$$
2 g(m+1) \rightarrow g^{\varepsilon}(m)+g^{-\varepsilon}(m+2) .
$$

We can choose a basis $e_{0}, \ldots, e_{m}, e_{0}^{\prime}, \ldots, e_{m}^{\prime}$ of $V$ such that $f\left(e_{r}, e_{s}\right)=f\left(e_{r}^{\prime}, e_{s}^{\prime}\right)=0$ and $f\left(e_{r}, e_{s}^{\prime}\right)=\delta_{r+s, m}$ for all $r, s$. We define the one-parameter family $x(t)=x_{0}+t x_{1}$ of operators in $V$ by

$$
\begin{aligned}
& x(t) e_{0}=e_{1}-\varepsilon(-1)^{m+m^{\prime}} t e_{0}^{\prime} \quad \text { if } m>0, \\
& x(t) e_{r}=e_{r+1} \quad(0<r<m), \quad x(t) e_{m}=-\varepsilon(-1)^{m^{\prime}} t e_{m}^{\prime}, \\
& x(t) e_{r}^{\prime}=-e_{r+1}^{\prime} \quad(0 \leqslant r<m), \quad x(t) e_{m}^{\prime}=0 .
\end{aligned}
$$

It is easy to verify that $x(t) \in L$ for all real $t$. Since $x_{0}$ consists of two Jordan blocks of size $m+1$ each, we have $x_{0} \in \mathcal{O}_{1}$. (Note that $m$ is odd if $j=6$ and $m$ is even if $j=9$.)

We have

$$
x(t)^{m+1} e_{0}=-2 \varepsilon(-1)^{m^{\prime}} t e_{m}^{\prime} \text { and }-\varepsilon i^{2 m^{\prime}} f\left(e_{0}, x(t)^{m+1} e_{0}\right)=2 t .
$$

Now let $t>0$. If $m=0$ then $x(t)$ is a single Jordan block and by (5.4)-(5.5) we have $x(t) \in \theta_{2}$. Now let $m \geqslant 1$. Then $x(t)^{m+2}=0$ and $\operatorname{dim} \operatorname{Ker} x(t)=2$. It follows that $x(t)$ consists of two Jordan blocks whose sizes are $m+2$ and $m$. The larger block is the type $\Delta_{m+1}^{-\varepsilon}(0)$ because of (12.3). To avoid tedious computations which are needed to determine the type of the smaller block, we shall use the following ad 
hoc argument. The smaller block is either of type $\Delta_{m-1}^{\varepsilon}(0)$ or of type $\Delta_{m-1}^{-\varepsilon}(0)$. When we let $t \rightarrow 0(t>0)$ the type must become $\Delta_{m-1}^{\varepsilon}(0)$. Otherwise there is a strictly decreasing sequence $t_{1}, t_{2}, \ldots$ with limit 0 for which the smaller block is of type $\Delta_{m-1}^{-\varepsilon}(0)$. By the necessity part of Theorem 5 , we then obtain $2 g(m+1) \leqslant$ $g^{-\varepsilon}(m+2)+g^{-\varepsilon}(m)$. By applying the priming operation $m-1$ times (see §6) we obtain $2 g(2) \leqslant g^{-\varepsilon}(3)+g^{-\varepsilon}(1)$, which is false. Hence for small positive $t$ the smaller block is of type $\Delta_{m-1}^{\varepsilon}(0)$. Consequently for small positive $t$ we have $x(t) \in \theta_{2}$. Since the set of all $t$ for which the smaller block is of type $\Delta_{m-1}^{\varepsilon}(0)$ (or $\left.\Delta_{m-1}^{-\varepsilon}(0)\right)$ is open, it follows that in fact $x(t) \in \theta_{2}$ for all $t>0$.

Next assume that (9.1) has the form

$$
g^{\varepsilon}(m+1)+g^{\varepsilon^{\prime}}(n+1) \rightarrow g^{\varepsilon}(m-1)+g^{\varepsilon^{\prime}}(n+3), \quad m \leqslant n .
$$

We can choose a basis $e_{0}, \ldots, e_{m}, e_{0}^{\prime}, \ldots, e_{n}^{\prime}$ of $V$ such that $f\left(e_{r}, e_{s}^{\prime}\right)=0$ for all $r, s$ and $f\left(e_{r}, e_{s}\right)=\varepsilon \delta_{r+s, m}$ and $f\left(e_{r}^{\prime}, e_{s}^{\prime}\right)=\varepsilon^{\prime} \delta_{r+s, n}$ for $r \leqslant s$. The family $x(t)$ is defined by

$$
\begin{aligned}
& x(t) e_{0}=e_{1}+\varepsilon t e_{0}^{\prime}, \\
& x(t) e_{r}=e_{r+1} \quad\left(0<r<m^{\prime}\right), \\
& x(t) e_{r}=-e_{r+1} \quad\left(m^{\prime} \leqslant r<m\right), \\
& x(t) e_{m}=0, \\
& x(t) e_{r}^{\prime}=e_{r+1}^{\prime} \quad\left(0 \leqslant r<n^{\prime}\right), \\
& x(t) e_{r}^{\prime}=-e_{r+1}^{\prime} \quad\left(n^{\prime} \leqslant r<n\right), \\
& x(t) e_{n}^{\prime}=-\varepsilon^{\prime} t e_{m} .
\end{aligned}
$$

One can easily verify that $x_{0}$ and $x_{1}$ are in $L$ and so $x(t) \in L$ for all real $t$. Moreover it is clear that $x_{0} \in \mathcal{O}_{1}($ see $\S 5)$. We have

$$
\begin{aligned}
& x(t)^{n+2} e_{0}=-\varepsilon \varepsilon^{\prime}(-1)^{n-n^{\prime}} t^{2} e_{m}, \quad \text { and } \\
& \varepsilon^{\prime}(-1)^{n-n^{\prime}+1} f\left(e_{0}, x(t)^{n+2} e_{0}\right)=t^{2} .
\end{aligned}
$$

Now let $t>0$. If $m=1$ then $x(t)$ is a single Jordan block and (12.5) and (5.4)-(5.5) show that it is of type $\Delta_{n+2}^{t^{\prime}}(0)$. Thus $x(t) \in \mathcal{O}_{2}$ in this case. Now let $m \geqslant 2$. Since $x(t)^{n+3}=0$ and $\operatorname{dim} \operatorname{Ker} x(t)=2, x(t)$ consists of two Jordan blocks whose sizes are $n+3$ and $m-1$. The larger block is of type $\Delta_{n+2}^{\varepsilon^{\prime}}(0)$, by (12.5) and (5.4)-(5.5). As in the case (12.2) we can conclude that for $t>0$ the other Jordan block of $x(t)$ must belong to the type $\Delta_{m-2}^{\varepsilon}(0)$. Thus $x(t) \in \mathcal{O}_{2}$ for $t>0$.

Now assume that the mutation (9.1) has the form

$$
\begin{aligned}
g^{\varepsilon}(m+1)+g^{+}(n+1)+g^{-}(n+1) & \\
& \rightarrow g^{\varepsilon}(m-1)+2 g(n+2), \quad m \leqslant n .
\end{aligned}
$$

We can choose a basis $e_{0}, \ldots, e_{n}, e_{0}^{\prime}, \ldots, e_{n}^{\prime}, e_{0}^{\prime \prime}, \ldots, e_{m}^{\prime \prime}$ of $V$ such that $f\left(e_{r}, e_{s}\right)=f\left(e_{r}^{\prime}, e_{s}^{\prime}\right)=0, f\left(e_{r}, e_{s}^{\prime \prime}\right)=f\left(e_{r}^{\prime}, e_{s}^{\prime \prime}\right)=0, f\left(e_{r}, e_{s}^{\prime}\right)=\delta_{r+s, n}$ for all $r, s$ and $f\left(e_{r}^{\prime \prime}, e_{s}^{\prime \prime}\right)=\varepsilon \delta_{r+s, m}$ for $r \leqslant s$. The family $x(t)$ of operators in $V$ is defined by

$$
\begin{aligned}
& x(t) e_{r}=e_{r+1} \quad(0 \leqslant r<n), \quad x(t) e_{n}=0, \\
& x(t) e_{r}^{\prime}=-e_{r+1}^{\prime} \quad(0 \leqslant r<n), \quad x(t) e_{n}^{\prime}=-\varepsilon t e_{m}^{\prime \prime},
\end{aligned}
$$




$$
\begin{aligned}
& x(t) e_{0}^{\prime \prime}=e_{1}^{\prime \prime}+t e_{0}, \\
& x(t) e_{r}^{\prime \prime}=e_{r+1}^{\prime \prime} \quad\left(0<r<m^{\prime}\right), \\
& x(t) e_{r}^{\prime \prime}=-e_{r+1}^{\prime \prime} \quad\left(m^{\prime} \leqslant r<m\right), \quad x(t) e_{m}^{\prime \prime}=0 .
\end{aligned}
$$

One can check that $x(t) \in L$ for all real $t$. Let $W, W^{\prime}$ and $W^{\prime \prime}$ be the subspaces of $V$ spanned by the vectors $e_{r}, e_{r}^{\prime}$ and $e_{r}^{\prime \prime}$, respectively. These subspaces are invariant under $x_{0}=x(0)$ and $W^{\prime \prime} \perp\left(W+W^{\prime}\right)$. The restriction of $x_{0}$ to each of these subspaces is just a single Jordan block. The block in $W^{\prime \prime}$ is of type $\Delta_{m}^{\varepsilon}(0)$. The other two subspaces, $W$ and $W^{\prime}$, are totally isotropic. This implies that the restriction of $x_{0}$ to $W+W^{\prime}$ belongs to the type $\Delta_{n}^{+}(0)+\Delta_{n}^{-}(0)$. Hence $x_{0} \in \theta_{1}$.

Now let $t \neq 0$. If $m=1$ one can easily check that $x(t)$ consists of two Jordan blocks of size $n+2$ and so $x(t) \in \mathcal{O}_{2}$. Now let $m \geqslant 2$. Let $V_{1}$ be the $x(t)$-invariant subspace generated by $e_{0}^{\prime}$. A basis of $V_{1}$ consists of the vectors $e_{r}^{\prime}(0 \leqslant r \leqslant n)$ and $e_{m}^{\prime \prime}$. Let $V_{2}$ be the $x(t)$-invariant subspace generated by $e_{0}^{\prime \prime}$. A basis of $V_{2}$ consists of the vectors $e_{0}^{\prime \prime}, \quad e_{r}^{\prime \prime}+t e_{r-1} \quad\left(1 \leqslant r \leqslant m^{\prime}\right), \quad(-1)^{r-m^{\prime}} e_{r}^{\prime \prime}+t e_{r-1} \quad\left(m^{\prime}<r \leqslant m\right), \quad e_{r}$ $(m \leqslant r \leqslant n)$. We have $V_{1} \cap V_{2}=0$ and $\operatorname{dim} V_{1}=\operatorname{dim} V_{2}=n+2$. Since $x(t)^{n+2}=0$ and

$$
x(t)^{m-2} e_{1}^{\prime \prime}=(-1)^{m-m^{\prime}-1} e_{m}^{\prime} \notin V_{1}+V_{2}, \quad x(t)^{m-1} e_{1}^{\prime \prime} \in V_{1},
$$

it follows that $x(t)$ consists of three Jordan blocks whose sizes are $n+2, n+2$, and $m-1$. Since $\varepsilon(-1)^{m-m^{\prime}-1} f\left(e_{1}^{\prime \prime}, x(t)^{m-2} e_{1}^{\prime \prime}\right)=1$, it follows that the Jordan block of size $m-1$ belongs to the type $\Delta_{m-2}^{\varepsilon}(0)$. Hence $x(t) \in \mathcal{O}_{2}$ for $t \neq 0$.

Next assume that (9.1) has the form

$$
g^{+}(m+1)+g^{-}(m+1)+g^{\varepsilon}(n+1) \rightarrow 2 g(m)+g^{\varepsilon}(n+3), \quad m \leqslant n .
$$

We can choose a basis $e_{0}, \ldots, e_{m}, e_{0}^{\prime}, \ldots, e_{m}^{\prime}, e_{0}^{\prime \prime}, \ldots, e_{n}^{\prime \prime}$ of $V$ such that $f\left(e_{r}, e_{s}\right)=f\left(e_{r}^{\prime}, e_{s}^{\prime}\right)=0, f\left(e_{r}, e_{s}^{\prime \prime}\right)=f\left(e_{r}^{\prime}, e_{s}^{\prime \prime}\right)=0, f\left(e_{r}, e_{s}^{\prime}\right)=\delta_{r+s, m^{\prime}}$ for all $r, s$ and $f\left(e_{r}^{\prime \prime}, e_{s}^{\prime \prime}\right)=\varepsilon \delta_{r+s, n}$ for $r \leqslant s$. We define the family $x(t)$ by

$$
\begin{aligned}
& x(t) e_{0}=e_{1}-\varepsilon t e_{0}^{\prime \prime} \quad\left(e_{1}=0 \text { if } m=0\right), \\
& x(t) e_{r}=e_{r+1} \quad(0<r<m), \\
& x(t) e_{m}=0 \quad \text { if } m>0, \\
& x(t) e_{r}^{\prime}=-e_{r+1}^{\prime} \quad(0 \leqslant r<m), \quad x(t) e_{m}^{\prime}=0, \\
& x(t) e_{r}^{\prime \prime}=e_{r+1}^{\prime \prime} \quad\left(0 \leqslant r<n^{\prime}\right), \\
& x(t) e_{r}^{\prime \prime}=-e_{r+1}^{\prime \prime} \quad\left(n^{\prime} \leqslant r<n\right), \quad x(t) e_{n}^{\prime \prime}=t e_{m}^{\prime} .
\end{aligned}
$$

One can check that $x(t) \in L$ for all real $t$. As in the case (12.6) one can show that $x_{0} \in \mathcal{O}_{1}$.

Now let $t \neq 0$. An easy computation gives

$$
\begin{aligned}
& x(t)^{n+2} e_{0}=(-1)^{n-n^{\prime}+1} \varepsilon t^{2} e_{m}, \quad \text { and } \\
& \varepsilon(-1)^{n-n^{\prime}+1} f\left(e_{0}, x(t)^{n+2} e_{0}\right)=t^{2} .
\end{aligned}
$$

If $m=0$ then (12.8) implies that $x(t) \in \vartheta_{2}$. Now let $m \geqslant 1$. Then it is easy to verify that $x(t)$ consists of three Jordan blocks whose sizes are $n+3, m$, and $m$. The 
largest block corresponds to the $x(t)$-invariant subspace generated by $e_{0}$. It follows from (12.8) that this block belongs to the type $\Delta_{m+2}^{\varepsilon}(0)$, and so $x(t) \in \mathcal{O}_{2}$ for $t \neq 0$.

Next assume that the mutation (9.1) has the form

$$
\begin{array}{r}
g^{+}(m+1)+g^{-}(m+1)+g^{+}(n+1)+g^{-}(n+1) \\
\rightarrow 2 g(m)+2 g(n+2), \quad m \leqslant n .
\end{array}
$$

We can choose a basis $a_{0}, \ldots, a_{m}, a_{0}^{\prime}, \ldots, a_{m}^{\prime}, b_{0}, \ldots, b_{n}, b_{0}^{\prime}, \ldots, b_{n}^{\prime}$ of $V$ such that $f\left(a_{r}, a_{s}\right)=f\left(a_{r}^{\prime}, a_{s}^{\prime}\right)=0, f\left(b_{r}, b_{s}\right)=f\left(b_{r}^{\prime}, b_{s}^{\prime}\right)=0, f\left(a_{r}, b_{s}\right)=f\left(a_{r}^{\prime}, b_{s}\right)=f\left(a_{r}, b_{s}^{\prime}\right)$ $=f\left(a_{r}^{\prime}, b_{s}^{\prime}\right)=0, f\left(a_{r}, a_{s}^{\prime}\right)=\delta_{r+s, m}$, and $f\left(b_{r}, b_{s}^{\prime}\right)=\delta_{r+s, n}$ for all $r, s$. We define the family $x(t)$ of operators in $V$ by

$$
\begin{aligned}
& x(t) a_{0}=a_{1}+t b_{0} \quad\left(a_{1}=0 \text { if } m=0\right), \\
& x(t) a_{r}=a_{r+1} \quad(0<r<m), \\
& x(t) a_{m}=0 \text { if } m>0 \text {, } \\
& x(t) a_{r}^{\prime}=-a_{r+1}^{\prime} \quad(0 \leqslant r<m), \quad x(t) a_{m}^{\prime}=0, \\
& x(t) b_{r}=b_{r+1} \quad(0 \leqslant r<n), \quad x(t) b_{n}=0, \\
& x(t) b_{r}^{\prime}=-b_{r+1}^{\prime} \quad(0 \leqslant r<n), \quad x(t) b_{n}^{\prime}=-t a_{m}^{\prime} .
\end{aligned}
$$

One can check that $x(t) \in L$ for all real $t$. By using the same argument as in the case (12.6) one can show that $x_{0} \in \mathcal{O}_{1}$.

Now let $t \neq 0$. Let $V_{1}$, resp. $V_{2}$, be the $x(t)$-invariant subspace generated by the vector $a_{0}$, resp. $b_{0}^{\prime}$. We have

$$
x(t)^{n+1} b_{0}^{\prime}=(-1)^{n} x(t) b_{n}^{\prime}=(-1)^{n+1} t a_{m}^{\prime}, \quad x(t)^{n+2} b_{0}^{\prime}=0 .
$$

For $m>0$ we have $x(t)^{n+1} a_{0}=x(t)^{n}\left(a_{1}+t b_{0}\right)=t x(t)^{n} b_{0}=t b_{n}$, and, for $m=0$, $x(t)^{n+1} a_{0}=t x(t)^{n} b_{0}=t b_{n}$. Thus for all $m$ we have $x(t)^{n+1} a_{0}=t b_{n}$ and $x(t)^{n+2} a_{0}=0$. It follows that $V_{1} \cap V_{2}=0$. If $m=0$ then $V=V_{1} \oplus V_{2}$, and so $x(t) \in \mathcal{O}_{2}$. Now let $m \geqslant 1$. Then $x(t)^{n+2}=0$ and $\operatorname{dim} \operatorname{Ker} x(t)=4$. Thus $x(t)$ consists of four Jordan blocks; the two largest of them arise from $V_{1}$ and $V_{2}$ and have size $n+2$. Since $x(t)^{m} V \subset V_{1}+V_{2}$, the other two Jordan blocks must be of size $m$ each. Therefore $x(t) \in \mathcal{O}_{2}$ for $t \neq 0$.

Next assume that the mutation (9.1) is of the form

$$
g^{\varepsilon}(m+1)+g^{-\varepsilon}(n+1) \rightarrow g^{-\varepsilon}(m-1)+g^{\varepsilon}(n+3), \quad m \leqslant n .
$$

We can choose a basis $e_{0}, \ldots, e_{m+k}, e_{0}^{\prime}, \ldots, e_{m+k}^{\prime}\left(k=n^{\prime}-m^{\prime}\right)$ of $V$ such that $f\left(e_{r}, e_{s}\right)=f\left(e_{r}^{\prime}, e_{s}^{\prime}\right)=0$ and $f\left(e_{r}, e_{s}^{\prime}\right)=\varepsilon(-1)^{n-m^{\prime}+1} \delta_{r+s, m+k}$ for all $r, s$. We define the family $x(t)$ of operators in $V$ by

$$
\begin{aligned}
& x(t) e_{r}=e_{r+1} \quad \text { for } r \neq k-1, k+1, k+m, \\
& x(t) e_{k-1}=e_{k}+(-1)^{m} e_{0}^{\prime} \quad \text { if } k>0, \\
& x(t) e_{k+1}=e_{k+2}+(-1)^{m} t e_{0}^{\prime} \quad\left(e_{k+2}=0 \text { if } m=1\right), \\
& x(t) e_{k+m}=-t e_{m-1}^{\prime}-e_{m+1}^{\prime} \quad\left(e_{m+1}^{\prime}=0 \text { if } k=0\right), \\
& x(t) e_{r}^{\prime}=-e_{r+1}^{\prime} \quad(0 \leqslant r<k+m), \quad x(t) e_{k+m}^{\prime}=0 .
\end{aligned}
$$

One can check that $x(t) \in L$ for all real $t$. If $k=0$ then $x_{0}$ consists of two Jordan blocks of size $m+1$. Since the subspaces spanned by $e_{0}, \ldots, e_{m+k}$ and $e_{0}^{\prime}, \ldots, e_{m+k}^{\prime}$ 
are $x_{0}$-invariant and totally isotropic, we have $x_{0} \in \mathcal{\vartheta}_{1}$. Now let $k \geqslant 1$. We have

$$
\begin{aligned}
& x_{0}^{n} e_{0}=2(-1)^{k} e_{k+m}^{\prime}, \quad x_{0}^{m}\left((-1)^{m} e_{0}^{\prime}-e_{k}\right)=e_{m}^{\prime}-e_{k+m}, \\
& x_{0}^{n+1} e_{0}=0, \quad x_{0}^{m+1}\left((-1)^{m} e_{0}^{\prime}-e_{k}\right)=0 .
\end{aligned}
$$

Hence $x_{0}$ consists of two Jordan blocks whose sizes are $n+1$ and $m+1$. Since

$$
-\varepsilon(-1)^{n-n^{\prime}} f\left(e_{0}, x_{0}^{n} e_{0}\right)=2, \quad \varepsilon(-1)^{m-m^{\prime}} f\left((-1)^{m} e_{0}^{\prime}-e_{k}, e_{m}^{\prime}-e_{k+m}\right)=2,
$$

we conclude that again $x_{0} \in \mathcal{O}_{1}$.

Now let $t>0$. A simple computation gives

$$
x(t)^{n+2} e_{0}= \begin{cases}2(-1)^{k} t e_{k+m}^{\prime} & \text { if } m>1, \\ (-1)^{k} t e_{k+m}^{\prime} & \text { if } m=1,\end{cases}
$$

and so

$$
\varepsilon(-1)^{n-n^{\prime}+1} f\left(e_{0}, x(t)^{n+2} e_{0}\right)>0 .
$$

Thus if $m=1$ then $x(t) \in \vartheta_{2}$ for $t>0$. Now let $m \geqslant 2$. Then $x(t)$ consists of two Jordan blocks of sizes $n+3$ and $m-1$. It follows from (12.11) that the larger block belongs to the type $\Delta_{n+2}^{\varepsilon}(0)$. The same argument as in (12.2) implies that the other block belongs to the type $\Delta_{m-2}^{-\varepsilon}(0)$. Therefore $x(t) \in \theta_{2}$ for $t>0$.

Next assume that the mutation (9.1) is of the form

$$
2 g^{\varepsilon}(m+1)+g^{-\varepsilon}(n+1) \rightarrow 2 g(m)+g^{\varepsilon}(n+3), \quad m<n .
$$

We can choose a basis $e_{0}, \ldots, e_{m+k}, e_{0}^{\prime}, \ldots, e_{m+k}^{\prime}, e_{0}^{\prime \prime}, \ldots, e_{m}^{\prime \prime}\left(k=n^{\prime}-m^{\prime}\right)$ of $V$ such that $f\left(e_{r}, e_{s}\right)=f\left(e_{r}^{\prime}, e_{s}^{\prime}\right)=0, f\left(e_{r}, e_{s}^{\prime \prime}\right)=f\left(e_{r}^{\prime}, e_{s}^{\prime \prime}\right)=0, f\left(e_{r}, e_{s}^{\prime}\right)=\varepsilon \delta_{r+s, k+m}$ for all $r, s$ and $f\left(e_{r}^{\prime \prime}, e_{s}^{\prime \prime}\right)=\varepsilon \delta_{r+s, m}$ for $r \leqslant s$. The family of operators $x(t)$ in $V$ is defined by

$$
\begin{aligned}
& x(t) e_{r}=e_{r+1} \quad \text { for } r \neq k-1, k+m-m^{\prime}, k+m, \\
& x(t) e_{k-1}=e_{k}-(-1)^{m^{\prime}} e_{0}^{\prime}, \\
& x(t) e_{k+m-m^{\prime}}=e_{k+m-m^{\prime}+1}+t e_{m-m^{\prime}}^{\prime \prime} \quad \text { if } m>0, \\
& x(t) e_{k+m}=(-1)^{m+m^{\prime}} e_{m+1}^{\prime} \quad \text { if } m>0, \\
& x(t) e_{k}=(-1)^{m+m^{\prime}} e_{m+1}^{\prime}+t e_{m-m^{\prime}}^{\prime \prime} \quad \text { if } m=0, \\
& x(t) e_{r}^{\prime}=-e_{r+1}^{\prime} \quad(0 \leqslant r<k+m), \quad x(t) e_{k+m}^{\prime}=0, \\
& x(t) e_{r}^{\prime \prime}=e_{r+1}^{\prime \prime} \quad\left(0 \leqslant r<m^{\prime}\right), \\
& x(t) e_{m^{\prime}}^{\prime \prime}=-t e_{m^{\prime}}^{\prime}-e_{m^{\prime}+1}^{\prime \prime} \quad\left(e_{m^{\prime}+1}^{\prime \prime}=0 \text { if } m=m^{\prime}\right), \\
& x(t) e_{r}^{\prime \prime}=-e_{r+1}^{\prime \prime} \quad\left(m^{\prime}<r<m\right), \quad x(t) e_{m}^{\prime \prime}=0 \quad \text { if } m>1 .
\end{aligned}
$$

One can check that $x(t) \in L$ for all real $t$. We have

$$
\begin{aligned}
& x_{0}^{n} e_{0}=-2(-1)^{m+n^{\prime}} e_{k+m}^{\prime}, \quad x_{0} e_{k+m}^{\prime}=0, \\
& -\varepsilon(-1)^{n-n^{\prime}} f\left(e_{0}, x_{0}^{n} e_{0}\right)=2 .
\end{aligned}
$$

If $a=e_{k}+(-1)^{m^{\prime}} e_{0}^{\prime}$ and $b=e_{k+m}+(-1)^{m+m^{\prime}} e_{m}^{\prime}$ then we find that

$$
x_{0}^{m} a=b, \quad x_{0} b=0, \quad \varepsilon(-1)^{m-m^{\prime}} f\left(a, x_{0}^{m} a\right)=2 .
$$


Note also that the restriction of $x_{0}$ to the subspace spanned by the vectors $e_{r}^{\prime \prime}$ is a Jordan block of type $\Delta_{m}^{\varepsilon}(0)$. Hence it follows from (12.13) and (12.14) that $x_{0} \in \mathcal{O}_{1}$.

Now let $t \neq 0$. An easy computation gives

$$
\text { 5) } x(t)^{n+2} e_{0}=-(-1)^{k+m-m^{\prime}} t^{2} e_{k+m}^{\prime}, \quad \varepsilon(-1)^{n-n^{\prime}+1} f\left(e_{0}, x(t)^{n+2} e_{0}\right)=t^{2} .
$$

Thus if $m=0$ then $x(t) \in \theta_{2}$. Now let $m \geqslant 1$. Then $x(t)^{n+3}=0$ and $\operatorname{dim} \operatorname{Ker} x(t)=3$. Thus $x(t)$ consists of three Jordan blocks. By (12.15) the largest block is of size $n+3$ and belongs to the type $\Delta_{n+2}^{\varepsilon}(0)$. Let $W$ be the $x(t)$-invariant subspace generated by $e_{0}$. Then $\operatorname{dim} W=n+3$ and $x(t)^{m} V \subset W$. This implies that the remaining two Jordan blocks are of size $m$ each. Therefore $x(t) \in \theta_{2}$ also when $m \geqslant 1$.

Now assume that the mutation (9.1) is of the form

$$
g^{\varepsilon}(m+1)+2 g^{-\varepsilon}(n+1) \rightarrow g^{-\varepsilon}(m-1)+2 g(n+2), \quad m<n .
$$

We can choose a basis $e_{0}, \ldots, e_{m+k}, e_{0}^{\prime}, \ldots, e_{m+k}^{\prime}, e_{0}^{\prime \prime}, \ldots, e_{n}^{\prime \prime}\left(k=n^{\prime}-m^{\prime}\right)$ of $V$ such that $f\left(e_{r}, e_{s}\right)=f\left(e_{r}^{\prime}, e_{s}^{\prime}\right)=0, f\left(e_{r}, e_{s}^{\prime \prime}\right)=f\left(e_{r}^{\prime}, e_{s}^{\prime \prime}\right)=0, f\left(e_{r}, e_{s}^{\prime}\right)=-\varepsilon \delta_{r+s, m+k}$ for all $r, s$ and $f\left(e_{r}^{\prime \prime}, e_{s}^{\prime \prime}\right)=-\varepsilon \delta_{r+s, n}$ for $r \leqslant s$. The family of operators $x(t)$ in $V$ is defined by

$$
\begin{aligned}
& x(t) e_{0}=e_{1}+2 t e_{0}^{\prime \prime} \quad \text { if } k>1, \\
& x(t) e_{0}=e_{1}-e_{0}^{\prime}+2 t e_{0}^{\prime \prime} \quad \text { if } k=1, \\
& x(t) e_{r}=e_{r+1} \text { for } r \neq 0, k-1, k+1, k+m, \\
& x(t) e_{k-1}=e_{k}+(-1)^{m^{\prime}} e_{0}^{\prime} \quad \text { if } k>1, \\
& x(t) e_{k+1}=e_{k+2}+2 t^{2}(-1)^{m^{\prime}} e_{0}^{\prime} \quad \text { if } m>1, \\
& x(t) e_{k+m}=-2 t^{2}(-1)^{m+m^{\prime}} e_{m-1}^{\prime}-(-1)^{m+m^{\prime}} e_{m+1}^{\prime} \quad \text { if } m>1, \\
& x(t) e_{k+m}=-4 t^{2} e_{0}^{\prime}-e_{2}^{\prime} \quad \text { if } m=1, \\
& x(t) e_{r}^{\prime}=-e_{r+1}^{\prime} \quad(0 \leqslant r<k+m), \quad x(t) e_{k+m}^{\prime}=0, \\
& x(t) e_{r}^{\prime \prime}=e_{r+1}^{\prime \prime} \quad\left(0 \leqslant r<n^{\prime}\right), \\
& x(t) e_{r}^{\prime \prime}=-e_{r+1}^{\prime \prime} \quad\left(n^{\prime} \leqslant r<n\right), \quad x(t) e_{n}^{\prime \prime}=-2 t e_{k+m}^{\prime} .
\end{aligned}
$$

Note that $x(t)$ depends quadratically on $t$, i.e., $x(t)=x_{0}+x_{1} t+x_{2} t^{2}$ where $x_{0}, x_{1}, x_{2}$ are constant operators and $x_{2} \neq 0$. One can check that $x(t) \in L$ for all real $t$.

We have $x_{0}^{n} e_{0}=2(-1)^{m+n^{\prime}} e_{k+m}^{\prime}, x_{0}^{n+1} e_{0}=0$, and

$$
-\varepsilon(-1)^{n-n^{\prime}} f\left(e_{0}, x_{0}^{n} e_{0}\right)=2 .
$$

If $a=e_{k}-(-1)^{m^{\prime}} e_{0}^{\prime}$ and $b=e_{k+m}-(-1)^{m+m^{\prime}} e_{m}^{\prime}$ then $x_{0}^{m} a=b, x_{0} b=0$, and

$$
\varepsilon(-1)^{m-m^{\prime}} f\left(a, x_{0}^{m} a\right)=2 .
$$

Note also that the restriction of $x_{0}$ to the subspace spanned by the vectors $e_{r}^{\prime \prime}$ is a Jordan block of type $\Delta_{n}^{-\varepsilon}(0)$. Hence it follows from (12.17) and (12.18) that $x_{0} \in \mathcal{O}_{1}$.

Now let $t \neq 0$. We have

$$
x(t)^{n+2}=0 \quad \text { and } \quad x(t)^{n+1} e_{0}=2 t(-1)^{m+n^{\prime}}\left(-2 t e_{m+k-1}^{\prime}+e_{n}^{\prime \prime}\right) .
$$


Hence $x(t)$ has a Jordan block of size $n+2$. But we know that such blocks must occur in pairs, and so there are two blocks of size $n+2$. Thus if $m=1$ then $x(t) \in \mathcal{O}_{2}$. Now let $m \geqslant 2$. Since $\operatorname{dim} \operatorname{Ker} x(t)=3, x(t)$ consists of three Jordan blocks. Hence the remaining Jordan block has size $m-1$. By invoking the necessity part of Theorem 5 we can conclude that this block must be of type $\Delta_{m-2}^{-\varepsilon}(0)$. Therefore $x(t) \in \mathcal{O}_{2}$.

Finally, assume that the mutation (9.1) has the form

$$
2 g^{\varepsilon}(m+1)+2 g^{-\varepsilon}(n+1) \rightarrow 2 g(m)+2 g(n+2), \quad m \leqslant n .
$$

Let $V=V_{1} \oplus V_{2}$ be an orthogonal decomposition with $V_{1}=W_{1} \oplus W_{1}^{\prime}$, $V_{2}=W_{2} \oplus W_{2}^{\prime}$ where $W_{1}, W_{1}^{\prime}, W_{2}, W_{2}^{\prime}$ are totally isotropic subspaces of dimension $m+k+1$ each $\left(k=n^{\prime}-m^{\prime}\right)$. We can choose bases $a_{0}, \ldots, a_{m+k} ; a_{0}^{\prime}, \ldots, a_{m+k}^{\prime}$; $b_{0}, \ldots, b_{m+k}$; and $b_{0}^{\prime}, \ldots, b_{m+k}^{\prime}$ of $W_{1}, W_{1}^{\prime}, W_{2}$, and $W_{2}^{\prime}$, respectively, such that $f\left(a_{r}, a_{s}^{\prime}\right)=f\left(b_{r}, b_{s}^{\prime}\right)=(-1)^{n-m^{\prime}+1} \varepsilon \delta_{r+s, m+k}$ for all $r, s$. Let $x_{0}$ be the operator in $V$ defined by

$$
\begin{aligned}
& x_{0} a_{r}=a_{r+1} \quad \text { for } r \neq k-1, k+m, \\
& x^{0} a_{k-1}=a_{k}+(-1)^{m} a_{0}^{\prime} \quad \text { if } k>0, \\
& x_{0} a_{k+m}=-a_{m+1}^{\prime} \quad\left(a_{m+1}^{\prime}=0 \text { if } k=0\right), \\
& x_{0} a_{r}^{\prime}=-a_{r+1}^{\prime} \quad(0 \leqslant r<m+k), \quad x_{0} a_{m+k}^{\prime}=0,
\end{aligned}
$$

and by analogous formulas obtained from these by replacing $a$ with $b$ everywhere. From the case (12.10) it follows that $x_{0} \in \mathcal{O}_{1}$. We make $V$ into a complex vector space by defining the action of $i$ by $i a_{r}=b_{r}$ and $i a_{r}^{\prime}=b_{r}^{\prime}$ for all $r$. We shall denote $V$ with this complex structure by $V^{\prime}$. Note that $a_{0}, \ldots, a_{m+k}, a_{0}^{\prime}, \ldots, a_{m+k}^{\prime}$ is a basis of $V^{\prime}$. There exists a unique hermitian form $f^{\prime}$ on $V^{\prime}$ such that $\operatorname{Re} f^{\prime}=f$ for $j=6$ (then $f$ is symmetric) and $\operatorname{Im} f^{\prime}=f$ for $j=9$ (then $f$ is skew-symmetric). Indeed $f^{\prime}$ is determined by

$$
\begin{array}{ll}
f^{\prime}\left(a_{r}, a_{s}\right)=f^{\prime}\left(a_{r}^{\prime}, a_{s}^{\prime}\right)=0, & \\
f^{\prime}\left(a_{r}, a_{s}^{\prime}\right)=(-1)^{n-m^{\prime}+1} \varepsilon \delta_{r+s, m+k} & \text { if } j=6, \\
f^{\prime}\left(a_{r}, a_{s}^{\prime}\right)=(-1)^{n-m^{\prime}+1}{ }_{i \varepsilon \delta_{r+s, m+k}} \text { if } j=9 .
\end{array}
$$

It is clear that $f^{\prime}$ is nondegenerate and $\operatorname{sig}\left(f^{\prime}\right)=(k+m, k+m)$. Let $U$ be the classical group of the fourth series defined by the pair $\left(V^{\prime}, f^{\prime}\right)$. Clearly $U$ is a subgroup of $G$ and we shall view its Lie algebra $M$ as a subalgebra of $L$. Note that $x_{0}$ is a complex linear operator in $V^{\prime}$ and that $x_{0} \in M$.

Let $Y_{1}=g^{\varepsilon}(m+1)+g^{-\varepsilon}(n+1)$ and $Y_{2}=g^{-\varepsilon}(m)+g^{\varepsilon}(n+2)$. Note that $\operatorname{sig} Y_{1}=\operatorname{sig} Y_{2}=(k+m, k+m)$. Let $\theta_{r}^{\prime}(r=1,2)$ be the nilpotent orbit of $U$ in $M$ corresponding to the chromosome $Y_{r}$. Note that $x_{0}$ as a complex linear operator consists of two Jordan blocks whose sizes are $n+1$ and $m+1$.

We claim that $x_{0} \in \mathcal{O}_{1}^{\prime}$. If $n=m$ this follows from the fact that $x_{0}$ has a totally isotropic Jordan block, for instance, its restriction to the subspace of $V^{\prime}$ spanned by the vectors $a_{r}$. Now let $k>0$. Then by using $x_{0}^{n} a_{0}=2(-1)^{k} a_{m+k}^{\prime}$ and (12.1) we find that $(-i)^{n}(-\varepsilon) f^{\prime}\left(a_{0}, x_{0}^{n} a_{0}\right)=2$, and so by (5.3) the larger block of $x_{0}$ is of type $\Delta_{n}^{-\varepsilon}(0)$. Similarly one can check that the other block is of type $\Delta_{m}^{\varepsilon}(0)$, and consequently 
$x_{0} \in \mathcal{O}_{1}^{\prime}$. This implies that $\theta_{1}^{\prime} \subset \theta_{1}$. On the other hand it is clear that $\theta_{2}^{\prime} \subset \theta_{2}$. Since $Y_{1} \leqslant Y_{2}$, we have $\theta_{1}^{\prime} \leqslant \theta_{2}^{\prime}$ by the case $j=4$ of Theorem 5. Consequently $\theta_{1} \leqslant \theta_{2}$.

Since every primitive $(2 \Lambda, \Pi)$ or $(\Pi, 2 \Lambda)$-mutation has one of the forms (12.2), (12.3), (12.6), (12.7), (12.9), (12.10), (12.12), (12.16), or (12.19), the proof of Theorem 5 is completed.

13. Closures of conjugacy classes. So far we have studied the closures of the orbits of a classical group $G$ in its Lie algebra $L$. It is not hard to transfer these results to the conjugacy classes of $G$. In this section we introduce the necessary concepts and state the main results. The proofs either follow from the case of orbits in $L$ or are obvious modifications of proofs already given, and so they will be omitted.

Each $x \in G$ has a unique multiplicative Jordan decomposition $x=x_{s} x_{u}$, where $x_{s}$ is semisimple, $x_{u}$ is unipotent, both $x_{s}$ and $x_{u}$ are in $G$, and $x_{s} x_{u}=x_{u} x_{s}$. We refer to $x_{s}\left(\right.$ resp. $x_{u}$ ) as the semisimple (resp. unipotent) component of $x$.

Recall that $G$ is a group of $\mathbf{F}$-linear transformations of a vector space $V$ which may be equipped with a form $f$ (see $\S 1$ ). We assume that it belongs to the $j$ th series. For $x \in G$ and $\lambda \in \mathbf{C}^{*}$ let

$$
V(\lambda, x)=\operatorname{Ker}(x-\lambda)^{\infty}
$$

if $\mathbf{F}=\mathbf{C}$. Otherwise let

$$
V(\lambda, x)=\operatorname{Ker}\left(x^{2}-2 \operatorname{Re}(\lambda) x+|\lambda|^{2}\right)^{\infty} .
$$

If $j \in\{5,8\}$ and $\lambda \neq \pm 1$ or $j \in\{4,6,7,9,10\}$ and $|\lambda| \neq 1$ then $V(\lambda, x)$ is totally isotropic. This leads us to define the subspace $V^{\prime}(\lambda, x)$ by

$$
V^{\prime}(\lambda, x)=\left\{\begin{array}{l}
V(\lambda, x) \text { for } j \in\{1,2,3\} \\
V(\lambda, x)+V\left(\lambda^{-1}, x\right) \text { for } j \in\{5,8\} \\
V(\lambda, x)+V\left(\bar{\lambda}^{-1}, x\right) \text { for } j \in\{4,6,7,9,10\} .
\end{array}\right.
$$

If $4 \leqslant j \leqslant 10$ the subspaces $V^{\prime}(\lambda, x)$ are nondegenerate and orthogonal to each other.

We obtain a direct (and orthogonal if $j \geqslant 4$ ) decomposition

$$
V=\sum_{\lambda \in \Omega_{j}^{*}} V^{\prime}(\lambda, x)
$$

where $\Omega_{j}^{*}$ is a subset of $\mathbf{C}^{*}$ specified by the Table V.

\begin{tabular}{c|l}
$j$ & \multicolumn{1}{|c}{$\Omega_{j}^{*}$} \\
\hline 1 & $\mathbf{C}^{*}$ \\
2,3 & $\operatorname{Im} \lambda \geqslant 0$ \\
4 & $|\lambda| \geqslant 1$ \\
5,8 & $\operatorname{Im} \lambda>0$ or $\left(\lambda \in \mathbf{R}^{*} \&|\lambda| \geqslant 1\right)$ \\
$6,7,9,10$ & $\operatorname{Im} \lambda \geqslant 0 \&|\lambda| \geqslant 1$
\end{tabular}


For $\lambda \in \mathbf{C}^{*}$ let $x_{\lambda}$ (resp. $\left.x_{\lambda}^{\prime}\right)$ be the restriction of $x$ to $V(\lambda, x)\left(\right.$ resp. $\left.V^{\prime}(\lambda, x)\right)$. If $1 \leqslant j \leqslant 3$ let $G(\lambda, x)$ be the general linear group of the space $V(\lambda, x)$. If $4 \leqslant j \leqslant 10$ let $G(\lambda, x)$ be the classical group defined by the subspace $V^{\prime}(\lambda, x)$ and the restriction of the form $f$ to this subspace. Thus in all cases $G(\lambda, x)$ is also a classical group of the $j$ th series. Clearly $x_{\lambda}^{\prime} \in G(\lambda, x)$ for all $\lambda$. We denote by $C(\lambda, x)$ the centralizer of $x_{\lambda}^{\prime}$ in $G(\lambda, x)$.

With this notation we have

$$
C_{G}(x)=\prod_{\lambda \in \Omega_{j}^{*}} C(\lambda, x) .
$$

The following analog of Theorem 1 is valid.

THEOREM 1 '. Let $G$ be a classical group of the jth series and $x \in G$ a semisimple element. Then each $C(\lambda, x)$ in (13.2) is a classical group of kth series where $k$ depends only on $j$ and $\lambda$ and is specified in the last column of Table III.

The group $G$ acts on itself by conjugation. We shall write $a \cdot x=a x a^{-1}$ for the result of the action of the element $a$ on $x \in G$. Then $G \cdot x=\left\{a x a^{-1}: a \in G\right\}$ is the conjugacy class of $x$ in $G$. The following analog of Theorem 2 is also valid.

TheOREM 2 . Let $G$ be a classical group, $x, y \in G$ and $x=x_{s} x_{u}$ the Jordan decomposition of $x$. Then $y \in \overline{G \cdot x}$ iff $a \cdot y=x_{s} z$ for some $a \in G$ and some

$$
z \in \overline{C_{G}\left(x_{s}\right) \cdot x_{u}} \text {. }
$$

This theorem reduces the problem of closures of conjugacy classes to the case of unipotent conjugacy classes. For this reason we shall now restrict our attention only to unipotent conjugacy classes.

Let $L$ be the Lie algebra of $G$ and exp: $L \rightarrow G$ the exponential map of $G$. It is known that exp induces a $G$-equivariant homeomorphism of the set of nilpotent elements of $L$ with the set of unipotent elements of $G$. (In particular, there is a bijection between the nilpotent orbits of $G$ in $L$ and the unipotent conjugacy classes of $G$.) This clearly solves the problem of closures of conjugacy classes of $G$.

14. Proof of Theorem 6: preliminaries. The remainder of this paper is devoted to the proof of Theorem 6. In this section we prove an important lemma (Lemma 9) and explain the method of proof.

It is convenient to represent chromosomes by diagrams. For instance the chromosome

$$
X=2 g(3)+g^{-}(2)+g(2)+g^{+}(4)
$$

can be represented by the diagram
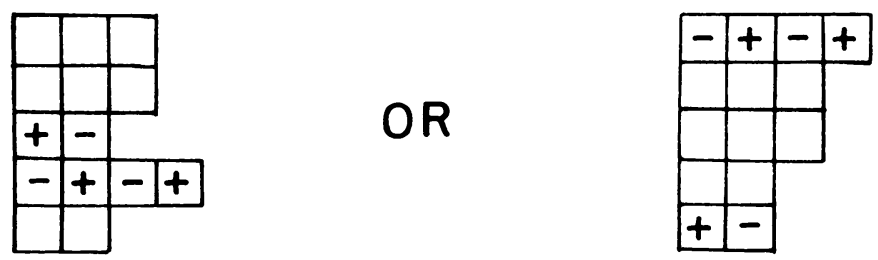
The rows of such diagrams can be permuted arbitrarily (they correspond to the constituent genes of $X$ ). We shall usually arrange rows in decreasing rank as in the second diagram above.

The priming operation on chromosomes was defined in $\S 6$. It is clear that the diagram of $X^{\prime}$ can be obtained from the diagram of $X$ by deleting its first column. The priming operation can be extended to varieties. Thus if $\Phi$ is a variety then $\Phi^{\prime}=\left\{X^{\prime}: X \in \Phi\right\}$ is also a variety. By iterating, we obtain the varieties $\Phi^{(k)}, k \geqslant 0$, where $\Phi^{(0)}=\Phi, \Phi^{(1)}=\Phi^{\prime}$, etc.

For instance we have $\Lambda^{\prime}=\Lambda, \Pi^{\prime}=\Pi,(2 \Lambda, \Lambda)^{\prime}=(\Lambda, 2 \Lambda),(\Lambda, 2 \Lambda)^{\prime}=(2 \Lambda, \Lambda)$, $(\Lambda, \Pi)^{\prime}=(\Pi, \Lambda), \quad(\Pi, \Lambda)^{\prime}=(\Lambda, \Pi), \quad(2 \Lambda, \Pi)^{\prime}=(\Pi, 2 \Lambda), \quad$ and $\quad(\Pi, 2 \Lambda)^{\prime}=$ $(2 \Lambda, \Pi)$.

The following lifting property of mutations will be often used in subsequent sections.

LeMma 9 (LIFTING PROPERTY). Let $\Phi$ be one of the varieties (6.2). If $X \in \Phi$ and $X^{(k)} \rightarrow U$ is a $\Phi^{(k)}$-mutation, then there exists a $\Phi$-mutation $X \rightarrow Z$ such that $Z^{(k)}=U$ and $\operatorname{sig} X^{(i)}=\operatorname{sig} Z^{(i)}$ for $0 \leqslant i \leqslant k$.

Proof. It suffices to consider the case when $X^{(k)} \rightarrow U$ is a primitive $\Phi^{(k)}$-mutation and $X^{(k)}$ has the same number of genes (counting multiplicities) as $X$. The rest of the proof consists in routine verifications.

We shall give the details only in the case when $X^{(k)} \rightarrow U$ is given by the formula (8.17), i.e.,

$$
X^{(k)}=g^{\varepsilon}(m)+2 g^{-\varepsilon}(n), \quad U=g^{-\varepsilon}(m-2)+2 g(n+1),
$$

where $1<m \leqslant n$ and $\varepsilon= \pm$. Note that $\Phi^{(k)}=(\Pi, 2 \Lambda)$ if $m$ and $n$ are even and $\Phi^{(k)}=(2 \Lambda, \Pi)$ when they are odd. (It is understood in (8.17) that $n-m$ is even.) Hence $\Phi=(\Pi, 2 \Lambda)$ if $m-k$ is even and $\Phi=(2 \Lambda, \Pi)$ if $m-k$ is odd. Since we assume that $X$ and $X^{(k)}$ have the same number of genes, we must have $X=$ $g^{\varepsilon}(m+k)+2 g^{-\varepsilon}(n+k)$. We claim that the $\Phi$-mutation $X \rightarrow Z$ where

$$
Z=g^{-\varepsilon}(m+k-2)+2 g(n+k+1)
$$

has the desired properties. Indeed it is easy to check that $\operatorname{sig} X^{(i)}=\operatorname{sig} Z^{(i)}$ for $0 \leqslant i \leqslant k$ and it is obvious that $Z^{(k)}=U$.

The $\Phi$-mutation $X \rightarrow Z$ having the properties mentioned in Lemma 9 will be called a lifting of the $\Phi^{(k)}$-mutation $X^{(k)} \rightarrow U$.

Let $\Phi$ be one of the varieties (6.2) and $X, Y \in \Phi$ such that $X<Y$ (i.e., $X \leqslant Y$ and $X \neq Y)$ and $\operatorname{sig} X=\operatorname{sig} Y$. In particular, $X$ and $Y$ have the same rank, say $n$, and so $X, Y \in \Phi(n)$. In order to prove Theorem 6 it suffices to prove the following statement:

(*) There exists a $\Phi$-mutation $X \rightarrow Z$ such that $Z \leqslant Y$.

The proof of this statement will be given by induction on the rank $n$. The verification of $(*)$ for small values of $n$ is straightforward and is omitted. The proof is divided into three sections: the next section treats the case $\Phi=\Pi$, the subsequent section the cases $\Phi=(\Pi, \Lambda)$ and $(\Lambda, \Pi)$, and the last one the cases $\Phi=(\Pi, 2 \Lambda)$ and $(2 \Lambda, \Pi)$. 
15. The case $\Phi=\Pi$. Before starting the proof we introduce some notations. To each $X \in \Pi$ we associate a $2 \times \infty$ nonnegative integral matrix $\sigma(X)$ defined by

$$
\sigma(X)=\left(\begin{array}{lll}
a_{0} & a_{1} & a_{2} \cdots \\
b_{0} & b_{1} & b_{2} \cdots
\end{array}\right)
$$

where $\left(a_{k}, b_{k}\right)=\operatorname{sig} X^{(k)}$ for all $k$. It is easy to see that the entries of this matrix satisfy the following conditions:

$$
\begin{gathered}
a_{0} \geqslant a_{1} \geqslant a_{2} \geqslant \ldots, \quad a_{k}=0 \quad \text { for large } k ; \\
b_{0} \geqslant b_{1} \geqslant b_{2} \geqslant \ldots, \quad b_{k}=0 \quad \text { for large } k ; \\
a_{0} \geqslant b_{1} \geqslant a_{2} \geqslant b_{3} \geqslant \ldots ; \\
\quad b_{0} \geqslant a_{1} \geqslant b_{2} \geqslant a_{3} \geqslant \ldots ; \\
a_{0}-a_{1} \geqslant b_{1}-b_{2} \geqslant a_{2}-a_{3} \geqslant b_{3}-b_{4} \geqslant \ldots ; \\
b_{0}-b_{1} \geqslant a_{1}-a_{2} \geqslant b_{2}-b_{3} \geqslant a_{3}-a_{4} \geqslant \ldots .
\end{gathered}
$$

The diagram of $X$ can be recovered from $\sigma(X)$ as follows: The first column of the diagram consists of $a_{0}-a_{1}$ positive and $b_{0}-b_{1}$ negative nodes; the second column consists of $a_{1}-a_{2}$ positive and $b_{1}-b_{2}$ negative nodes, etc. Hence the map $\sigma$ is a bijection between $\Pi$ and the set of all $2 \times \infty$ integral matrices whose entries satisfy the conditions (15.2)-(15.7).

Let $X, Y \in \Pi(n)$ satisfy $X<Y$. We shall show that there exists a $\Pi$-mutation $X \rightarrow Z$ such that $Z \leqslant Y$. We use induction on $n$. For $n=0,1$ the assertion is vacuously true; so let $n>1$. Let $\sigma(X)$ be as above and let

$$
\sigma(Y)=\left(\begin{array}{lll}
c_{0} & c_{1} & c_{2} \\
d_{0} & d_{1} & d_{2}
\end{array}\right)
$$

Since $X<Y$, we have

$$
a_{k} \leqslant c_{k}, \quad b_{k} \leqslant d_{k} \quad(k \geqslant 0) .
$$

Since $a_{0}+b_{0}=n=c_{0}+d_{0}$, it follows from (15.8) that $a_{0}=c_{0}$ and $b_{0}=d_{0}$.

Assume that $X$ and $Y$ are not disjoint, i.e., there is a gene $g$ such that $X=X_{1}+g$ and $Y=Y_{1}+g$, with $X_{1}, Y_{1} \in \Pi$. Then $X_{1}<Y_{1}$ and by induction hypothesis there exists a $\Pi$-mutation $X_{1} \rightarrow Z_{1}$ such that $Z_{1} \leqslant Y_{1}$. Then $X \rightarrow Z=Z_{1}+g$ is also a $\Pi$-mutation and $Z \leqslant Y$. Thus we may assume (and we do) that $X$ and $Y$ are disjoint.

Assume now that $\left(a_{k}, b_{k}\right)=\left(c_{k}, d_{k}\right) \neq(0,0)$ for some $k \geqslant 1$. Since $X<Y$, we have $X^{(k)} \leqslant Y^{(k)}$. Since $X$ and $Y$ are disjoint, so are $X^{(k)}$ and $Y^{(k)}$ and consequently $X^{(k)}<Y^{(k)}$. By induction hypothesis there exists a $\Pi$-mutation $X^{(k)} \rightarrow U$ such that $U \leqslant Y^{(k)}$. By the lifting property (Lemma 9) there exists a $\Pi$-mutation $X \rightarrow Z$ such that $Z^{(k)}=U$ and $Z \leqslant Y$. Hence from now on we may also assume that

$$
Y^{(k)} \neq 0 \& k \geqslant 1 \Rightarrow a_{k}<c_{k} \text { or } b_{k}<d_{k} .
$$

Now assume that $X \supset g^{+}(k)+g^{-}(k)$ for some $k$. Then $Y$ contains no genes of rank $k$. Since $X<Y$, we have $Y^{(k)} \neq 0$. By (15.9) we have $a_{k}<c_{k}$ or $b_{k}<d_{k}$. We define a $\Pi$-mutation $X \rightarrow Z$ by $g^{+}(k)+g^{-}(k) \rightarrow g^{\epsilon}(k+1)+g^{-\varepsilon}(k-1)$, where 
$\varepsilon=+$ if $a_{k}<c_{k}$ and $\varepsilon=-$ otherwise. Clearly $Z \leqslant Y$. Hence we may assume from now on (and we do) that

$$
X \not \supset g^{+}(k)+g^{-}(k), \quad k \geqslant 1 .
$$

Until further notice we shall assume that $a_{1}<c_{1}$. Let $g_{1}=g_{\varepsilon_{1}}(m)$ be a gene of $X$ with $m$ minimal.

Case 1: $\varepsilon_{1}=-$. Since $a_{1}<c_{1}, X$ contains a gene of the form $g_{2}=g_{+}(k)$. Assume also that $k$ is minimal. We define a $\Pi$-mutation $X \rightarrow Z$ by $g_{1}+g_{2} \rightarrow$ $g_{-}(k+1)+g_{+}(m-1)$. If $k$ is odd we have

$$
\begin{aligned}
c_{k-1}-c_{k} & \leqslant d_{k-2}-d_{k-1} \leqslant \cdots \leqslant c_{0}-c_{1}<a_{0}-a_{1} \\
& =b_{1}-b_{2}=\cdots=b_{k-2}-b_{k-1}=a_{k-1}-a_{k} .
\end{aligned}
$$

Therefore $a_{k}<c_{k}, b_{k-1}<d_{k-1}, a_{k-2}<c_{k-2}, \ldots, b_{2}<d_{2}$ and so $Z \leqslant Y$. If $k$ is even we have $d_{k-1}-d_{k} \leqslant c_{k-2}-c_{k-1} \leqslant \cdots \leqslant c_{0}-c_{1}<a_{0}-a_{1}=b_{1}-b_{2}=\cdots$ $=a_{k-2}-a_{k-1}=b_{k-1}-b_{k}$. Hence $b_{k}<d_{k}, a_{k-1}<c_{k-1}, b_{k-2}<d_{k-2}, \ldots, b_{2}<$ $d_{2}$, and again we have $Z \leqslant Y$.

Case 2: $g_{1}=g_{+}(1)$. Since $b_{0}=c_{0} \geqslant c_{1}>a_{1}, X$ must contain a negative gene. Let $g_{2}=g^{-}(k)$ be such a gene with $k$ minimal. Define a $\Pi$-mutation $X \rightarrow Z$ by $g_{1}+g_{2} \rightarrow g^{+}(k+1)$. For $1 \leqslant i \leqslant k$ we have

$$
d_{0}-d_{i-1} \geqslant c_{1}-c_{i}>a_{1}-c_{i}=\left(a_{1}-a_{i}\right)+\left(a_{i}-c_{i}\right)=b_{0}-b_{i-1}+\left(a_{i}-c_{i}\right) .
$$

Since $b_{0}=d_{0}$ and $b_{i-1} \leqslant d_{i-1}$, we infer that $a_{i}<c_{i}$, and so $Z \leqslant Y$.

Case 3: $\varepsilon_{1}=+, m \geqslant 2$, and $X \supset 2 g_{1}$. We define the $\Pi$-mutation $X \rightarrow Z$ by $2 g_{1} \rightarrow g_{+}(m-2)+g_{+}(m+2)$. We have $b_{1}-b_{2}=a_{0}-a_{1}>c_{0}-c_{1} \geqslant d_{1}-d_{2}$, and so $b_{2}<d_{2}$. Let $m$ be even and $i=m-1$ or $m$. Then $c_{1}-c_{i} \leqslant d_{0}-d_{i-1} \leqslant b_{0}$ $-b_{i-1}=a_{1}-a_{i}$ and $d_{2}-d_{i+1} \leqslant d_{0}-d_{i-1} \leqslant b_{0}-b_{i-1}=b_{2}-b_{i+1}$. Consequently, $a_{i}<c_{i}$ and $b_{i+1}<d_{i+1}$, and so $Z \leqslant Y$. Now let $m$ be odd and $i=m-1$ or $m$. Then $c_{1}-c_{i+1} \leqslant d_{0}-d_{i} \leqslant b_{0}-b_{i}=a_{1}-a_{i+1}$ and $d_{2}-d_{i} \leqslant d_{0}-d_{i-2} \leqslant b_{0}$ $-b_{i-2}=b_{2}-b_{i}$. Since $a_{1}<c_{1}$ and $b_{2}<d_{2}$, it follows that $a_{i+1}<c_{i+1}$ and $b_{i}<d_{i}$. Thus we have again $Z \leqslant Y$.

Case 4: $\varepsilon_{1}=+, m \geqslant 2$, and $X \not \supset 2 g_{1}$. Clearly $X^{(m)} \neq 0$, and let $g_{2}=g^{\varepsilon_{2}}(k)$ be a gene of $X$ with $k$ minimal subject to $k>m$. Note that $g_{1}=g^{\varepsilon}(m)$ where $\varepsilon=(-1)^{m-1}$.

Let first $\varepsilon_{2}=-\varepsilon$. Then we define a $\Pi$-mutation $X \rightarrow Z$ by $g_{1}+g_{2} \rightarrow g^{\varepsilon}(k+1)+$ $g^{-\varepsilon}(m-1)$. As in Case 3 we have $a_{m}<c_{m}$ and $b_{m}<d_{m}$. Let $m$ be even and $m \leqslant i \leqslant k-1$. For $i$ even we have $d_{i}-d_{i+1} \leqslant d_{0}-d_{1} \leqslant b_{0}-b_{1}=b_{i}-b_{i+1}$, and for $i$ odd

$$
\begin{aligned}
d_{i}-d_{i+1} & \leqslant d_{1}-d_{2} \leqslant c_{0}-c_{1} \leqslant a_{0}-a_{1}-1 \\
& =b_{m-1}-b_{m}-1=a_{m}-a_{m+1}=b_{i}-b_{i+1} .
\end{aligned}
$$

Since $b_{m}<d_{m}$, we obtain $b_{i}<d_{i}(m \leqslant i \leqslant k)$, and so $Z \leqslant Y$. Now let $m$ be odd and $m \leqslant i \leqslant k-1$. For $i$ even we have

$$
c_{i}-c_{i+1} \leqslant c_{0}-c_{1} \leqslant a_{0}-a_{1}-1=a_{m-1}-a_{m}-1=b_{m}-b_{m+1}=a_{i}-a_{i+1},
$$

and for $i$ odd $c_{i}-c_{i+1} \leqslant c_{1}-c_{2} \leqslant d_{0}-d_{1} \leqslant b_{0}-b_{1}=a_{i}-a_{i+1}$. Since $a_{m}<c_{m}$, we obtain $a_{i}<c_{i}(m \leqslant i \leqslant k)$, and so we have again $Z \leqslant Y$. 
Now let $\varepsilon_{2}=\varepsilon$ and let $k-m$ be even. Then we define a $\Pi$-mutation $X \rightarrow Z$ by $g_{1}+g_{2} \rightarrow g^{\varepsilon}(k+2)+g^{\varepsilon}(m-2)$. As in Case 3 we have $a_{m-1}<c_{m-1}, a_{m}<c_{m}$, $b_{m}<d_{m}$ and, if $m>2, b_{m-1}<d_{m-1}$. Let $m$ be even and $m \leqslant i \leqslant k-1$. For $i$ even we have

$$
c_{i}-c_{i+1} \leqslant c_{0}-c_{1} \leqslant a_{0}-a_{1}-1=a_{m}-a_{m+1}=a_{i}-a_{i+1},
$$

and for $i$ odd $c_{i}-c_{i+1} \leqslant c_{1}-c_{2} \leqslant d_{0}-d_{1} \leqslant b_{0}-b_{1}=a_{i}-a_{i+1}$. It follows that $a_{i}<c_{i}$ for $m-1 \leqslant i \leqslant k$. The proof of $b_{i}<d_{i}(m \leqslant i \leqslant k+1)$ is the same as when $\varepsilon_{2}=-\varepsilon$ except that one has to use also the equality $a_{k-1}-a_{k}=b_{k}-b_{k+1}$. Thus $Z \leqslant Y$. Now let $m$ be odd and $m \leqslant i \leqslant k-1$. For $i$ even we have $d_{i}-d_{i+1} \leqslant d_{0}-d_{1} \leqslant b_{0}-b_{1}=b_{i}-b_{i+1}$, and for $i$ odd

$$
d_{i}-d_{i+1} \leqslant c_{0}-c_{1} \leqslant a_{0}-a_{1}-1=b_{m}-b_{m+1}=b_{i}-b_{i+1} .
$$

Thus $b_{i}<d_{i}$ for $m-1 \leqslant i \leqslant k$. The proof of $a_{i}<c_{i}(m \leqslant i \leqslant k+1)$ is the same as when $\varepsilon_{2}=-\varepsilon$ except that one has to use also the equality $b_{k-1}-b_{k}=a_{k}-a_{k+1}$. Hence again $Z \leqslant Y$.

Finally let $\varepsilon_{2}=\varepsilon$ and let $k-m$ be odd. Then we define the $\Pi$-mutation $X \rightarrow Z$ by $g_{1}+g_{2} \rightarrow g^{\varepsilon}(k+1)+g^{\varepsilon}(m-1)$. The proof of $Z \leqslant Y$ is the same as when $k-m$ is even.

This completes the Case 4 and exhausts all possibilities when $a_{1}<c_{1}$. If $b_{1}<d_{1}$ then by interchanging positive and negative charges in both $X$ and $Y$, we reduce this case to the case $a_{1}<c_{1}$. In view of (15.9) this completes the proof.

16. The cases $\Phi=(\Pi, \Lambda)$ or $(\Lambda, \Pi)$. We shall treat the two cases simultaneously. All mutations in this proof are understood to be $\Phi$-mutations. Let $X, Y \in \Phi(n)$ and $X<Y$. We shall show that there exists a mutation $X \rightarrow Z$ such that $Z \leqslant Y$. The proof is by induction on $n$. For $n=0,1$ the assertion is vacuously true, so let $n>1$. We shall write $\operatorname{sig} X^{(i)}=\left(a_{i}, b_{i}\right)$, sign $Y^{(i)}=\left(c_{i}, d_{i}\right), r_{i}=a_{i}+b_{i}, s_{i}=c_{i}+d_{i}$. Since $X<Y$, we have $\left(a_{i}, b_{i}\right) \leqslant\left(c_{i}, d_{i}\right)$ for all $i$. Since $a_{0}+b_{0}=n=c_{0}+d_{0}$, we have $a_{0}=c_{0}$ and $b_{0}=d_{0}$. If $\Phi=(\Lambda, \Pi)(\operatorname{resp} ., \Phi=(\Pi, \Lambda))$ then $a_{i}=b_{i}$ and $c_{i}=d_{i}$ for $i$ odd (resp., $i$ even), and $a_{i}, b_{i}, c_{i}, d_{i}$ are integers for $i$ even (resp. $i$ odd). As in the previous section we may assume that $X$ and $Y$ are disjoint. In view of the lifting property and our induction hypothesis, we may assume (and we do) that

$$
Y^{(i)} \neq 0 \& i \geqslant 1 \Rightarrow r_{i}<s_{i} .
$$

Assume that $X \supset g^{+}(m)+g^{-}(m)$ for some $m$. We define the mutation $X \rightarrow Z$ by $g^{+}(m)+g^{-}(m) \rightarrow g(m-1)+g(m+1)$. Since $X$ and $Y$ are disjoint, we have $Y^{(m)} \neq 0$. Then (16.1) implies that $r_{m}<s_{m}$, and consequently $Z \leqslant Y$. Hence from now on we may assume that $X \not \supset g^{+}(m)+g^{-}(m), m \geqslant 1$.

Next assume that $X \supset 2 g(m)$ for some $m$. Since $X$ and $Y$ are disjoint and $X<Y$, we have $Y^{(m)} \neq 0$. By (16.1) we have $a_{m}<c_{m}$ or $b_{m}<d_{m}$. Then we have $X \rightarrow Z \leqslant Y$ where the mutation $X \rightarrow Z$ is defined by $2 g(m) \rightarrow g^{-\varepsilon}(m-1)+$ $g^{\varepsilon}(m+1) ; \varepsilon=+$ if $a_{m}<c_{m}$ and $\varepsilon=-$ if $a_{m}=c_{m}$. Without loss of generality we assume from now on that $X \not \supset 2 g(m), m \geqslant 1$.

From now on let $g_{1}$ be a gene of $X$ of minimum rank $r\left(g_{1}\right)=m$. 
When $g_{1}$ is nonpolarized we denote by $g_{2}$ a gene of $X-g_{1}$ of minimum rank $r\left(g_{2}\right)=k$. Since $g_{1}=g(m)$ and $X \not \supset 2 g(m)$, we have $m<k$. Then we distinguish two cases.

Case 1: $g_{2}=g(k)$. By (16.1) $r_{m}<s_{m}$, and so $a_{m}<c_{m}$ or $b_{m}<d_{m}$. By switching + and - charges in all polarized genes of $X$ and $Y$ (if necessary) we may assume that $a_{m}<c_{m}$. If $i-m$ is even and $m \leqslant i \leqslant k-2$ then

$$
c_{i}-c_{i+2} \leqslant s_{i}-s_{i+1} \leqslant s_{0}-s_{1} \leqslant r_{0}-r_{1}-1=r_{i}-r_{i+1}=a_{i}-a_{i+2} .
$$

Since $a_{m}<c_{m}$, these inequalities imply that $a_{j}<c_{j}$ for $m \leqslant j \leqslant k$. Hence we have $X \rightarrow Z \leqslant Y$, where $X \rightarrow Z$ is defined by $g(m)+g(k) \rightarrow g^{-}(m-1)+g^{+}(k+1)$.

Case 2: $g_{2}$ is polarized. Without loss of generality we may assume that $g_{2}=g^{+}(k)$. If $i-m$ is even and $m \leqslant i \leqslant k-3$ then $d_{i}-d_{i+2} \leqslant s_{i}-s_{i+1} \leqslant s_{0}-s_{1} \leqslant r_{0}-r_{1}$ $-1=r_{i}-r_{i+1}=b_{i}-b_{i+2}$. If $m \geqslant 2$ we have

$$
d_{m-2}-d_{m} \leqslant s_{m-2}-s_{m-1} \leqslant s_{0}-s_{1}<r_{0}-r_{1}=r_{m-2}-r_{m-1}=b_{m-2}-b_{m-1} \text {, }
$$

and so $b_{m}<d_{m}$. Thus if $m \geqslant 2$ or if $m=1$ and $b_{1}<d_{1}$, it follows that $b_{j}<d_{j}$ for $m \leqslant j \leqslant k-1$. Under these conditions we have $X \rightarrow Z \leqslant Y$, where $X \rightarrow Z$ is defined by $g(m)+g^{+}(k) \rightarrow g^{+}(m-1)+g(k+1)$. Now let $m=1$ and $b_{1}=d_{1}$. Then $c_{1}-a_{1}=s_{1}-r_{1} \geqslant 1$ and consequently $X-g_{1}-g_{2}$ must contain either a negative or a nonpolarized gene. Let $g_{3}$ be such a gene of minimal rank $r\left(g_{3}\right)=t$. If $i$ is even and $2 \leqslant i \leqslant t-1$ then

$$
c_{i}-c_{i+1} \leqslant c_{0}-c_{1} \leqslant a_{0}-a_{1}-1<b_{1}-b_{2}=a_{2}-a_{3}=\cdots=a_{i}-a_{i+1} .
$$

Hence $a_{j}<c_{j}$ for $1 \leqslant j \leqslant t$. If $t$ is even then $g_{3}=g^{-}(t)$ and we have $X \rightarrow Z \leqslant Y$, with $X \rightarrow Z$ defined by $g_{2}+g_{3} \rightarrow g(k-1)+g(t+1)$. If $t$ is odd then $g_{3}=g(t)$ and we have $X \rightarrow Z \leqslant Y$, with $X \rightarrow Z$ defined by $g_{2}+g_{3} \rightarrow g(k-1)+g^{+}(t+1)$.

Now assume that $g_{1}$ is polarized. Without any loss of generality we assume that $g_{1}=g^{+}(m)$. We now distinguish several cases.

Case 3: $m \geqslant 3$. We have

$$
\begin{aligned}
& c_{m-1}-c_{m+1} \leqslant s_{m-1}-s_{m} \leqslant s_{0}-s_{1}<r_{0}-r_{1}=a_{m-1}-a_{m+1}, \\
& d_{m-3}-d_{m-1} \leqslant s_{m-3}-s_{m-2} \leqslant s_{0}-s_{1}<r_{0}-r_{1}=b_{m-3}-b_{m-1},
\end{aligned}
$$

and so $a_{m+1}<c_{m+1}$ and $b_{m-1}<d_{m-1}$. If $X \supset 2 g_{1}$ then we have $X \rightarrow Z \leqslant Y$, where $X \rightarrow Z$ is defined by $2 g_{1} \rightarrow g^{+}(m-2)+g^{+}(m+2)$. We may now assume that $X \not \supset 2 g_{1}$, and let $g_{2}$ be a gene of $X-g_{1}$ of minimal rank $r\left(g_{2}\right)=k$. If $i-m$ is odd and $m+1 \leqslant i \leqslant k-2$, we have $c_{i}-c_{i+2} \leqslant s_{i}-s_{i+1} \leqslant s_{0}-s_{1} \leqslant r_{0}-r_{1}-1=r_{i}$ $-r_{i+1}=a_{i}-a_{i+2}$. It follows that $a_{j}<c_{j}$ for $m \leqslant j \leqslant k$. If $g_{2}=g^{+}(k)$ we also obtain by the same argument that $c_{k-1}-c_{k+1} \leqslant a_{k-1}-a_{k+1}$, and $a_{k+1}<c_{k+1}$. If $i-m$ is odd and $m-1 \leqslant i \leqslant k-2$, we have

$$
d_{i}-d_{i+2} \leqslant s_{i}-s_{i+1} \leqslant s_{0}-s_{1} \leqslant r_{0}-r_{1}-1=r_{i+1}-r_{i+2}=b_{i}-b_{i+2} .
$$

Since $b_{m-1}<d_{m-1}$, we infer that $b_{j}<d_{j}$ for $m-1 \leqslant j \leqslant k$. Hence we have $X \rightarrow Z \leqslant Y$, where $X \rightarrow Z$ is defined by

$$
\begin{aligned}
& g_{1}+g_{2} \rightarrow g(m-1)+g^{+}(k+1) \quad \text { if } g_{2}=g(k) ; \\
& g_{1}+g_{2} \rightarrow g(m-1)+g(k+1) \quad \text { if } g_{2}=g^{-}(k) ; \\
& g_{1}+g_{2} \rightarrow g^{+}(m-2)+g^{+}(k+2) \quad \text { if } g_{2}=g^{+}(k) .
\end{aligned}
$$


Case 4: $m=2$. Since $c_{1}-c_{3} \leqslant s_{1}-s_{2} \leqslant s_{0}-s_{1}<r_{0}-r_{1}=a_{1}-a_{3}$ and $a_{1} \leqslant c_{1}$, we have $a_{3}<c_{3}$. If $b_{1}<d_{1}$ then we can proceed exactly as in Case 1 . Thus we may assume that $b_{1}=d_{1}$, and so $a_{1}<c_{1}$. Then $X$ contains a negative or a nonpolarized gene. Let $g_{2}$ be such a gene of minimal rank $r\left(g_{2}\right)=k$. If $i$ is even and $2 \leqslant i \leqslant k-1$, we have $a_{i}-a_{i+1}=b_{i-1}-b_{i}=\cdots=a_{0}-a_{1}>c_{0}-c_{1} \geqslant c_{i}-c_{i+1}$. Hence $a_{j}<c_{j}$ for $1 \leqslant j \leqslant k$ and we have $X \rightarrow Z \leqslant Y$, where $X \rightarrow Z$ is defined by

$$
\begin{array}{ll}
g_{1}+g_{2} \rightarrow g(1)+g^{+}(k+1) & \text { if } g_{2}=g(k) ; \\
g_{1}+g_{2} \rightarrow g(1)+g(k+1) & \text { if } g_{2}=g^{-}(k) .
\end{array}
$$

Case 5: $m=1$. We have now $a_{1}=b_{1}<c_{1}=d_{1}$. Therefore $X$ contains a negative or a nonpolarized gene. Let $g_{2}$ be such a gene of minimal rank $r\left(g_{2}\right)=k$. If $i$ is even and $2 \leqslant i \leqslant k$ then

$$
a_{i-1}-a_{i}=b_{i-2}-b_{i-1}=\cdots=b_{0}-b_{1}>d_{0}-d_{1} \geqslant c_{1}-c_{2} \geqslant \cdots \geqslant c_{i-1}-c_{i} .
$$

It follows that $a_{j}<c_{j}$ for $1 \leqslant j \leqslant k$. Then we have $X \rightarrow Z \leqslant Y$, where $X \rightarrow Z$ is defined by

$$
\begin{array}{ll}
g_{1}+g_{2} \rightarrow g^{+}(k+1) & \text { if } g_{2}=g(k) ; \\
g_{1}+g_{2} \rightarrow g(k+1) & \text { if } g_{2}=g^{-}(k) .
\end{array}
$$

17. The cases $\Phi=(\Pi, 2 \Lambda)$ or $(2 \Lambda, \Pi)$. All mutations in this proof will be $\Phi$-mutations. We treat the two cases simultaneously. Let $X, Y \in \Phi(n)$ and $X<Y$. We have to show that there exists a mutation $X \rightarrow Z$ such that $Z \leqslant Y$. We use induction on $n$. If $n=0,1$ then the assertion is vacuously true; so let $n>1$. Let $\left(a_{i}, b_{i}\right)=\operatorname{sig} X^{(i)}$ and $\left(c_{i}, d_{i}\right)=\operatorname{sig} Y^{(i)}$, and let $r_{i}=a_{i}+b_{i}, s_{i}=c_{i}+d_{i}$. Note that $\left(a_{i}, b_{i}\right) \leqslant\left(c_{i}, d_{i}\right), r_{0}=n=s_{0}$, and $a_{0}=c_{0}, b_{0}=d_{0}$. Furthermore, if $\Phi=(2 \Lambda, \Pi)$ then $a_{i}=b_{i}$ and $c_{i}=d_{i}$ for $i$ odd, while if $\Phi=(\Pi, 2 \Lambda)$ then $a_{i}=b_{i}$ and $c_{i}=d_{i}$ for $i$ even.

As in $\S 16$ we may assume that $X$ and $Y$ are disjoint. In view of the lifting property we may also assume that

$$
Y^{(i)} \neq 0 \& i \geqslant 1 \Rightarrow r_{i}<s_{i} .
$$

Assume that $X \supset 2 g(m)$ for some $m$. Since $X$ and $Y$ are disjoint and $X<Y$, we have $Y^{(m)} \neq 0$ and by (17.1) $a_{m}<c_{m}$ or $b_{m}<d_{m}$. Define $X \rightarrow Z$ by $2 g(m) \rightarrow$ $g^{\varepsilon}(m+1)+g^{-\varepsilon}(m-1)$ where $\varepsilon=+$ if $a_{m}<c_{m}$ and $\varepsilon=-$ if $a_{m}=c_{m}$. Then $Z \leqslant Y$. Hence from now on we may assume (and we do) that $X$ is polarized.

Hence all inequalities (15.2)-(15.7) are valid. By replacing each nonpolarized pair $2 g(m)$ in $Y$ by $g^{+}(m)+g^{-}(m)$ we obtain a polarization $U$ of $Y$. Since $\operatorname{sig} U^{(i)}=\left(c_{i}, d_{i}\right)$, it follows that the $c_{i}$ 's and the $d_{i}$ 's satisfy the analogs of the inequalities (15.2)-(15.7). In the sequel we shall use freely these inequalities without explicit reference to them.

Let us assume that $X \supset g^{+}(m)+g^{-}(m)$ for some $m$. Assume also that $m$ is minimal with respect to this property. Since $Y^{(m)} \neq 0$ and $s_{m}$ and $r_{m}$ are even, it follows from (17.1) that $s_{m}-r_{m} \geqslant 2$.

If $X \supset 2 g^{+}(m)+2 g^{-}(m)$ then $X \rightarrow Z \leqslant Y$ where $X \rightarrow Z$ is defined by $2 g^{+}(m)+2 g^{-}(m) \rightarrow 2 g(m-1)+2 g(m+1)$. 
Now let $X \supset 2 g^{\varepsilon}(m)+g^{-\varepsilon}(m)$ and $X \not \supset 2 g^{-\varepsilon}(m)$. By switching + and - signs in $X$ and $Y$ (if necessary) we may assume that $\varepsilon=+$. If $Y^{(m+1)} \neq 0$ we can choose $\varepsilon_{1}$ so that $r^{\varepsilon_{1}}\left(X^{(m+1)}\right)<r^{\varepsilon_{1}}\left(Y^{(m+1)}\right)$. Then $X \rightarrow Z \leqslant Y$ where $X \rightarrow Z$ is defined by $2 g^{+}(m)+g^{-}(m) \rightarrow 2 g(m-1)+g^{\varepsilon_{1}}(m+2)$. Now let $Y^{(m+1)}=0$. Since $X$ and $Y$ are disjoint and $X<Y$, we have $Y \supset 2 g(m+1)$, and so $b_{m-1}=1<d_{m-1}$. Then $X \rightarrow Z \leqslant Y$ where $X \rightarrow Z$ is defined by $2 g^{+}(m)+g^{-}(m) \rightarrow g^{+}(m-2)+$ $2 g(m+1)$.

Now let $m \geqslant 2, X \not \supset 2 g^{+}(m)$, and $X \not \supset 2 g^{-}(m)$. Then $a_{m-1}<c_{m-1}$ or $b_{m-1}<$ $d_{m-1}$. Without any loss of generality we may assume that $b_{m-1}<d_{m-1}$. If $b_{m+1}<$ $d_{m+1}$ then $X \rightarrow Z \leqslant Y$ with $X \rightarrow Z$ defined by $g^{+}(m)+g^{-}(m) \rightarrow g^{+}(m-2)+$ $g^{-}(m+2)$. Now let $b_{m+1}=d_{m+1}$. Then $a_{m-1}-a_{m}-1=b_{m}-b_{m+1}=b_{m}-d_{m+1}<$ $d_{m}-d_{m+1} \leqslant c_{m-1}-c_{m}$, and so $c_{m-1}-a_{m-1}>\left(c_{m}-a_{m}\right)-1 \geqslant 0$. If $a_{m+1}<$ $c_{m+1}$ then $X \rightarrow Z \leqslant Y$ where $X \rightarrow Z$ is defined by $g^{+}(m)+g^{-}(m) \rightarrow g^{-}(m-2)+$ $g^{+}(m+2)$. Now let also $a_{m+1}=c_{m+1}$. Then by (17.1) $Y^{(m+1)}=0$, and so $Y \supset$ $2 g(m+1)$. Let $g^{\varepsilon}(k)$ be a gene of $X-g^{+}(m)-g^{-}(m)$ of maximal rank. Clearly $k<m$ and, by minimality of $m, X \not \supset g^{-\varepsilon}(k)$. This implies that $k \geqslant 2$. Then we have $X \rightarrow Z \leqslant Y$ where $X \rightarrow Z$ is defined by $g^{f}(k)+g^{+}(m)+g^{-}(m) \rightarrow g^{f}(k-2)+$ $2 g(m+1)$.

Finally let $m=1, X \not \supset 2 g^{+}(1)$, and $X \not \supset 2 g^{-}(1)$. Then $Y$ has no genes of rank one. Thus $X-g^{+}(1)-g^{-}(1)$ is nonempty, and let $g^{\varepsilon}(k)$ be its gene of minimal rank. Without loss of generality we may assume (and we do) that $\varepsilon=+$. We have

$$
a_{0}-a_{2}=b_{0}-b_{2}=r_{0}-r_{1}-1>s_{0}-s_{1}=c_{0}-c_{2}=d_{0}-d_{2},
$$

and so $a_{2}<c_{2}$ and $b_{2}<d_{2}$. If $i$ is even and $2 \leqslant i \leqslant k-3$, then

$$
\begin{aligned}
& a_{i}-a_{i+2}=r_{i}-r_{i+1}=r_{0}-r_{1}-2 \geqslant s_{0}-s_{1} \geqslant s_{i}-s_{i+1} \geqslant c_{i}-c_{i+2}, \\
& b_{i}-b_{i+2}=r_{i}-r_{i+1} \geqslant s_{i}-s_{i+1} \geqslant d_{i}-d_{i+2},
\end{aligned}
$$

and consequently $a_{j}<c_{j}$ and $b_{j}<d_{j}$ for $1 \leqslant j \leqslant k$.

If $X \supset g^{-}(k)$ then we have $X \rightarrow Z \leqslant Y$, where $X \rightarrow Z$ is defined by $g^{+}(1)+g^{-}(1)+g^{+}(k)+g^{-}(k) \rightarrow 2 g(k+1)$.

Otherwise we have

$$
a_{k-1}-a_{k+1}=r_{k-1}-r_{k}=r_{0}-r_{1}-2 \geqslant s_{0}-s_{1} \geqslant c_{k-1}-c_{k+1},
$$

and so $a_{k+1}<c_{k+1}$. Now we have $X \rightarrow Z \leqslant Y$ where $X \rightarrow Z$ is defined by $g^{+}(1)+g^{-}(1)+g^{+}(k) \rightarrow g^{+}(k+2)$.

Hence from now on we may assume (and we do) that $X \not \supset g^{+}(m)+g^{-}(m)$, $m \geqslant 1$,

In the remaining part of the proof let $m$ be the minimum rank of a gene of $X$. We assume (as we may) that $X \supset g^{+}(m)$, and consequently $X \not \supset g^{-}(m)$. We now distinguish several cases.

Case 1: $m \geqslant 3$. From $a_{m-1}-a_{m+1}=r_{m-1}-r_{m}=r_{0}-r_{1}>s_{0}-s_{1} \geqslant s_{m-1}-s_{m}$ $\geqslant c_{m-1}-c_{m+1}$ and $b_{m-3}-b_{m-1}=r_{0}-r_{1}>s_{0}-s_{1} \geqslant d_{m-3}-d_{m-1}$ it follows that $a_{m+1}<c_{m+1}$ and $b_{m-1}<d_{m-1}$. If $X \supset 2 g^{+}(m)$ then we have $X \rightarrow Z \leqslant Y$ where $X \rightarrow Z$ is defined by $2 g^{+}(m) \rightarrow g^{+}(m-2)+g^{+}(m+2)$. Now assume that $X$ $\not \supset 2 g^{+}(m)$ and let $g^{\varepsilon}(k)$ be a gene of $X-g^{+}(m)$ of minimum rank. Clearly 
$k \geqslant m+2$. If $i-m$ is odd and $m+1 \leqslant i \leqslant k-3$ then

$$
a_{i}-a_{i+2}=r_{i}-r_{i+1}=r_{0}-r_{1}-1 \geqslant s_{0}-s_{1} \geqslant s_{i}-s_{i+1} \geqslant c_{i}-c_{i+2} .
$$

If $\varepsilon=+$ this is also valid for $i=k-1$. Since $a_{m+1}<c_{m+1}$, we conclude that $a_{j} \leqslant c_{j}$ holds for $m \leqslant j \leqslant k$ and also for $j=k+1$ if $\varepsilon=+$. If $i-m$ is odd and $m-1 \leqslant i \leqslant k-3$ then $b_{i}-b_{i+2}=r_{0}-r_{1}-1 \geqslant s_{0}-s_{1} \geqslant d_{i}-d_{i+2}$. If $\varepsilon=-$ this is also valid for $i=k-1$. Since $b_{m-1}<d_{m-1}$, we conclude that $b_{j}<d_{j}$ holds for $m-1 \leqslant j \leqslant k$ and also for $j=k+1$ if $\varepsilon=-$. Hence we have $X \rightarrow Z \leqslant Y$ where $X \rightarrow Z$ is defined by $g^{+}(m)+g^{\varepsilon}(k) \rightarrow g^{+}(m-2)+g^{\varepsilon}(k+2)$.

Case 2: $m=2$. Since $a_{1}-a_{3}=r_{1}-r_{2}=r_{0}-r_{1}>s_{0}-s_{1} \geqslant s_{1}-s_{2} \geqslant c_{1}-c_{3}$ and $a_{1} \leqslant c_{1}$, we have $a_{3}<c_{3}$. If $b_{1}<d_{1}$ we can proceed exactly as in Case 1 . Thus we may assume that $b_{1}=d_{1}$, and consequently $a_{1}<c_{1}$. It follows that $X \supset g^{-}(k)$ for some $k$. Clearly $k \geqslant 4$ and we may assume that $k$ is minimal. If $i$ is even and $2 \leqslant i \leqslant k-2$ we have

$$
a_{i}-a_{i+1}=b_{i-1}-b_{i}=\cdots=a_{0}-a_{1}>c_{0}-c_{1} \geqslant c_{i}-c_{i+1},
$$

and so $c_{i+1}-a_{i+1}>c_{i}-a_{i} \geqslant 1$. Hence $c_{j}-a_{j} \geqslant 2$ for $j$ odd satisfying $3 \leqslant j \leqslant k-1$. If $a_{k+1}<c_{k+1}$ then we have $X \rightarrow Z \leqslant Y$ where $X \rightarrow Z$ is defined by $g^{+}(2)+g^{-}(k) \rightarrow g^{+}(k+2)$. Now let $a_{k+1}=c_{k+1}$. Since

$$
b_{k-1}-b_{k}=a_{0}-a_{1}>c_{0}-c_{1} \geqslant c_{k}-c_{k+1}=c_{k}-a_{k+1}>a_{k}-a_{k+1} \text {, }
$$

we have $X \supset 2 g^{-}(k)$. Now we have $X \rightarrow Z \leqslant Y$ where $X \rightarrow Z$ is defined by $g^{+}(2)+2 g^{-}(k) \rightarrow 2 g(k+1)$.

Case 3: $m=1$ and $X \supset 2 g^{+}(1)$. Since $a_{1}=b_{1}<c_{1}=d_{1}$, we have $X \supset g^{-}(k)$ for some $k$. Assume that $k$ is minimal; of course $k \geqslant 3$. If $i$ is even and $2 \leqslant i \leqslant k-1$ we have

$$
a_{i-1}-a_{i}=b_{i-2}-b_{i-1}=\cdots=b_{0}-b_{1}>d_{0}-d_{1} \geqslant c_{1}-c_{2} \geqslant \cdots \geqslant c_{i-1}-c_{i},
$$

and so $c_{i}-a_{i}>c_{i-1}-a_{i-1} \geqslant 1, c_{i}-a_{i} \geqslant 2$. If $a_{k+1}<c_{k+1}$ then we have $X \rightarrow Z$ $\leqslant Y$ where $X \rightarrow Z$ is defined by $2 g^{+}(1)+g^{-}(k) \rightarrow g^{+}(k+2)$. Now let $a_{k+1}=c_{k+1}$. Since $b_{k-1}-b_{k}=b_{0}-b_{1}>d_{0}-d_{1} \geqslant c_{k}-c_{k+1}=c_{k}-a_{k+1}>a_{k}-a_{k+1}$, we have $X \supset 2 g^{-}(k)$. Thus we have $X \rightarrow Z \leqslant Y$ where $X \rightarrow Z$ is defined by $2 g^{+}(1)+$ $2 g^{-}(k) \rightarrow 2 g(k+1)$.

Case 4: $m=1$ and $X \not \supset 2 g^{+}(1)$. Since $s_{1}-r_{1} \geqslant 2$, we have $r_{0}-r_{1} \geqslant s_{0}-s_{1}+2$. In particular, $X$ has at least three genes. Let us write

$$
X=g^{+}(1)+g^{\varepsilon}(k)+g^{\varepsilon_{1}}(t)+X_{1}, \quad 3 \leqslant k \leqslant t,
$$

where $X_{1} \in \Phi$ has no genes of rank less than $t$. We define a mutation $X \rightarrow Z$ by

$$
g^{\varepsilon}(k)+g^{\varepsilon_{1}}(t) \rightarrow g^{\varepsilon}(k-2)+g^{\varepsilon_{1}}(t+2) .
$$

We have

$$
\begin{aligned}
a_{k-3}-a_{k-1} & =r_{k-3}-r_{k-2} \geqslant r_{0}-r_{1}-1>s_{0}-s_{1} \\
& \geqslant s_{k-3}-s_{k-2} \geqslant c_{k-3}-c_{k-1}, \\
b_{k-3}-b_{k-1} & =r_{k-2}-r_{k-1}=r_{0}-r_{1}-1>s_{0}-s_{1} \\
& \geqslant s_{k-3}-s_{k-2} \geqslant d_{k-3}-d_{k-1},
\end{aligned}
$$

and so $a_{k-1}<c_{k-1}$ and $b_{k-1}<d_{k-1}$. 
If $k=t$ we have $\varepsilon_{1}=\varepsilon$. Then

$$
\begin{aligned}
r^{\varepsilon}\left(X^{(k-1)}-X^{(k+1)}\right) & =r_{k-1}-r_{k}=r_{0}-r_{1}-1>s_{0}-s_{1} \\
& \geqslant s_{k-1}-s_{k} \geqslant r^{\varepsilon}\left(Y^{(k-1)}-Y^{(k+1)}\right) .
\end{aligned}
$$

Since $r^{\varepsilon}\left(X^{(k-1)}\right)<r^{\varepsilon}\left(Y^{(k-1)}\right)$, we infer that also $r^{\varepsilon}\left(X^{(k+1)}\right)<r^{\varepsilon}\left(Y^{(k+1)}\right)$ and so $Z \leqslant Y$.

Now let $k<t$. Let $i-k$ be odd and $k-1 \leqslant i \leqslant t-3$. Then

$$
\begin{aligned}
& a_{i}-a_{i+2} \geqslant r_{k}-r_{k+1}=r_{0}-r_{1}-2 \geqslant s_{0}-s_{1} \geqslant s_{i}-s_{i+1} \geqslant c_{i}-c_{i+2}, \\
& b_{i}-b_{i+2} \geqslant r_{k}-r_{k+1} \geqslant d_{i}-d_{i+2} .
\end{aligned}
$$

If $\varepsilon_{1}=+$ then by the same argument we find that $a_{t-1}-a_{t+1} \geqslant c_{t-1}-c_{t+1}$, while if $\varepsilon_{1}=-$ we obtain $b_{t-1}-b_{t+1} \geqslant d_{t-1}-d_{t+1}$. Since $a_{k-1}<c_{k-1}$ and $b_{k-1}<d_{k-1}$, these inequalities imply that $Z \leqslant Y$.

This completes the proof of Theorem 6 .

\section{REFERENCES}

1. N. Bourgoyne and R. Cushman, Conjugacy classes in linear groups, J. Algebra 44 (1977), 339-362.

2. J. Dixmier, Polarisations dans les algèbres de Lie semisimples complexes, Bull. Sci. Math. (2) 99 (1975), $45-63$.

3. D. Ž. Djoković, Closures of conjugacy classes in the classical complex Lie groups, Houston J. Math. 6 (1980), 245-257.

4. Closures of conjugacy classes in classical real linear Lie groups, Algebra, Carbondale 1980 (Proceedings), Lecture Notes in Math., vol. 848, Springer-Verlag, Berlin and New York.

5. M. Gerstenhaber, On dominance and varieties of commuting matrices, Ann. of Math. (2) 73 (1961), $324-348$.

6. , Dominance over the classical groups, Ann of Math. (2) 74 (1961), 532-569.

7. W. Hesselink, Singularies in the nilpotent scheme of a classical group, Trans. Amer. Math. Soc. 222 (1976), 1-32.

Department of Mathematics, University of Waterloo, Waterloo, Ontario, Canada N2L 3Gl

Current address: Department of Mathematics, Rutgers University, Hill Center, New Brunswick, New Jersey 08903 